\title{
ON REPRESENTATIONS AND INTEGRABILITY OF MATHEMATICAL STRUCTURES IN ENERGY-CONSERVING PHYSICAL SYSTEMS*
}

\author{
MORTEN DALSMO ${ }^{\dagger}$ AND ARJAN VAN DER SCHAFT ${ }^{\ddagger}$
}

\begin{abstract}
In the present paper we elaborate on the underlying Hamiltonian structure of interconnected energy-conserving physical systems. It is shown that a power-conserving interconnection of port-controlled generalized Hamiltonian systems leads to an implicit generalized Hamiltonian system, and a power-conserving partial interconnection to an implicit port-controlled Hamiltonian system. The crucial concept is the notion of a (generalized) Dirac structure, defined on the space of energy-variables or on the product of the space of energy-variables and the space of flow-variables in the port-controlled case. Three natural representations of generalized Dirac structures are treated. Necessary and sufficient conditions for closedness (or integrability) of Dirac structures in all three representations are obtained. The theory is applied to implicit port-controlled generalized Hamiltonian systems, and it is shown that the closedness condition for the Dirac structure leads to strong conditions on the input vector fields.
\end{abstract}

Key words. Hamiltonian systems, Dirac structures, implicit systems, external variables, integrability, actuated mechanical systems, kinematic constraints, interconnections

AMS subject classifications. 93C10, 93A30, 70F25, 58F05

PII. S0363012996312039

1. Introduction. Most of the current modelling and simulation approaches to (complex) physical systems (e.g., multibody systems) are based on some sort of network representation, where the physical system under consideration is seen as the interconnection of a (possible large) number of simple subsystems. This way of modelling has several advantages. From a physical point of view it is usually natural to regard the system as composed of subsystems, possibly from different domains (mechanical, electrical, and so on). The knowledge about subsystems can be stored in libraries, and is reusable for later occasions. Because of the modularity the modelling process can be performed in an "iterative" manner, gradually refining - if necessarythe model by adding other subsystems. Further, the approach is suited to general control design where the overall behavior of the system is sought to be improved by the addition of other subsystems or controlling devices. From a system-theoretic point of view this modular approach naturally emphasizes the need for models of systems with external variables, e.g., inputs and outputs.

In this paper we concentrate on the mathematical description of network representations of (lumped-parameter) energy-conserving physical systems. In our previous work we have shown how energy-conserving physical systems with independent energy variables can be naturally described as generalized Hamiltonian systems (with external variables). However, a general power-conserving interconnection of such systems will lead to a system described by differential and algebraic equations, that is, an implicit dynamical system, which can no longer be directly described as an explicit

${ }^{*}$ Received by the editors November 13, 1996; accepted for publication (in revised form) January 22, 1998; published electronically September 25, 1998.

http://www.siam.org/journals/sicon/37-1/31203.html

$\dagger$ ABB Corporate Research, Department of Information Technology and Control Systems, P.O. Box 90, N-1361 Billingstad, Norway (morten.dalsmo@nocrc.abb.no).

$\ddagger$ University of Twente, Systems and Control Group, Department of Applied Mathematics, 7500 AE Enschede, The Netherlands (A.J.vanderSchaft@math.utwente.nl). 
generalized Hamiltonian system. This motivates the definition of implicit generalized Hamiltonian systems, as introduced in [SM2, SM3]. The main ingredient in this definition is that of a (generalized) Dirac structure. The relevance of Dirac structures in the Hamiltonian modelling of electrical LC-circuits with dependent storage elements (a clear example of interconnected energy-conserving systems) was already recognized in [C2].

The notion of Dirac structures was introduced by Courant and Weinstein [CW] and further investigated by Courant in [C1] as a generalization of Poisson and (pre)symplectic structures. Dorfman [D1, D2] developed an algebraic theory of Dirac structures in the context of the study of completely integrable systems of partial differential equations, with the aim of describing within a Hamiltonian framework certain sets of PDEs which do not admit an easy Hamiltonian formulation in terms of Poisson or symplectic structures, due to nonlocality of the involved operators. The conceptual novelty in the approach initiated in [C2, SM2, SM3] is to use Dirac structures for the direct Hamiltonian description of differential-algebraic equations resulting from the interconnection of energy-conserving systems, including constrained systems. Although the terminology Dirac structure is derived from the "Dirac bracket" introduced by Dirac in his study of constrained Hamiltonian systems arising from degenerate Lagrangians [D3], our use of Dirac structures determining, together with the stored energy (Hamiltonian), the algebraic constraints as well as the dynamical equations of motion seems to be new. Furthermore, we stress the "physical" relevance of Dirac structures as naturally capturing the geometric structure of the system as arising from the interconnection of subsystems (see e.g., Proposition 2.2).

In Courant and Dorfman [C1, D2] the definition of a Dirac structure includes a closedness (or integrability) condition generalizing the Jacobi-identity for Poisson brackets or the closedness of two-forms defining symplectic structures. This condition is naturally satisfied for constant Dirac structures (as in the case of LC-circuits) and for Dirac structures arising from holonomic kinematic constraints in mechanical systems, but not for the generalized Dirac structures arising from nonholonomic kinematic constraints [SM1, SM3] or from general kinematic pairs in multibody systems [M2].

The structure of this paper is as follows. In section 2 we will recall the definitions of a (generalized) Dirac structure and of an implicit Hamiltonian system, and we will show how the power-conserving interconnection of port-controlled (explicit) Hamiltonian systems leads to such an implicit Hamiltonian system. In section 3 we will investigate various useful ways of representing generalized Dirac structures and consequently of representing implicit Hamiltonian systems, and we will study their relationship. Then in section 4 the closedness (or integrability) condition for Dirac structures will be worked out for the three different representations obtained. Both sections 3 and 4 use extensively techniques and results from the work of Courant and Dorfman, although the emphasis is rather different. The results of sections 3 and 4 are applied in section 5 to Dirac structures as arising in implicit generalized Hamiltonian systems with external variables. In particular it is shown that the closedness condition translates into strong conditions on the input vector fields.

A main motivation for the Hamiltonian modelling of interconnected energy-conserving physical systems is, apart from the clear motivation from a general modelling and simulation point of view, the generalization of the theory of "passivity-based control" to complex interconnected physical systems. Key concepts in this theory (see, e.g., [TA, OS, S]) are the use of the internal energy as candidate Lyapunov function, the shaping of the internal energy via state feedback, and the injection of "damping" in order to achieve asymptotic stability. This approach has shown to be very powerful 
in the robust and/or adaptive control of physical systems described by Euler-Lagrange or Hamiltonian equations of motion (such as robot manipulators, mobile robots, and electrical machines) and can be expected to be equally powerful for interconnected physical systems. Although it is not the topic of the present paper to demonstrate this, we indicate at the end of section 4 how the usual stability theory of Hamiltonian systems based on the Hessian matrix of the Hamiltonian can be naturally extended to implicit Hamiltonian systems. Moreover, at the end of section 5 we show the link between results in this paper and "passivity-based control" of actuated mechanical systems with kinematic constraints.

In the control design of interconnected physical systems also the system-theoretic properties (such as controllability and observability) of implicit port-controlled Hamiltonian systems will prove to be instrumental (e.g., in the analysis how much damping injection is needed for asymptotic stabilization). For explicit port-controlled generalized Hamiltonian systems some of these topics already have been studied in our previous work [SM2, MS1, MS2]. Section 5 provides only a basic framework for a study of these issues. Apart from "passivity-based control", the further exploitation of the structure of symmetries and conservation laws also has a great potential (see, e.g., [BKMM] for related developments). All this is a large area for further research.

2. Generalized Hamiltonian modelling of interconnected systems. In our previous work [MS1, MS2, MBS, MSB1, MSB2, SM1, SM2, SM3] we have argued that the basic dynamic building blocks in the network representation of energyconserving physical systems are systems of the form

$$
\begin{aligned}
\dot{x} & =J(x) \frac{\partial H}{\partial x}(x)+g(x) f, \\
e & =g^{T}(x) \frac{\partial H}{\partial x}(x) .
\end{aligned}
$$

Here $x=\left(x_{1}, \ldots, x_{n}\right)$ denotes the vector of (independent) energy variables, defining local coordinates for the state space manifold $\mathcal{X}, H\left(x_{1}, \ldots, x_{n}\right)$ is the total stored energy in the system, with $\frac{\partial H}{\partial x}(x)$ denoting the column-vector of partial derivatives of $H$, and the $n \times n$ skew-symmetric structure matrix $J(x)$ is associated with the network topology of the system. The columns $g_{j}(x), j=1, \ldots, m$, of the matrix $g(x)$ define the (state modulated) transformers describing the influence of the external flow sources (or inputs) $f_{j}, j=1, \ldots, m$. The components $e_{j}$ of $e$ are the corresponding conjugated (with respect to the power) efforts (or outputs). Since the matrix $J(x)$ is skew-symmetric we immediately obtain the energy balance

$$
\frac{\mathrm{d}}{\mathrm{dt}} H=e^{T} f
$$

expressing that the increase in energy equals the externally supplied power $\left(e_{j} f_{j}\right.$ is the power of the $j$ th source). Thus (2.1) describes an energy-conserving physical system with internal variables $x_{1}, \ldots, x_{n}$ (associated with energy storage) and external (or port) variables $f_{1}, \ldots, f_{m}, e_{1}, \ldots, e_{m}$ (associated with power), which can be regarded, respectively, as input and output variables.

The system (2.1) is called a port-controlled generalized Hamiltonian system because of the following. We may define a generalized Poisson bracket operation on the real functions on $\mathcal{X}$ as

$$
\{F, G\}(x)=\left[\frac{\partial F}{\partial x}(x)\right]^{T} J(x) \frac{\partial G}{\partial x}(x), \quad F, G: \mathcal{X} \rightarrow \mathbb{R},
$$


Clearly, this bracket is skew-symmetric and satisfies the Leibniz identity

$$
\left\{F, G_{1} G_{2}\right\}(x)=\left\{F, G_{1}\right\}(x) G_{2}(x)+G_{1}(x)\left\{F, G_{2}\right\}(x) \text { for all } F, G_{1}, G_{2}: \mathcal{X} \rightarrow \mathbb{R}
$$

and thus $\dot{x}=J(x) \frac{\partial H}{\partial x}(x)$ can be seen as the generalized Hamiltonian vector field corresponding to $H$ and the generalized Poisson bracket $\{$,$\} . This generalized Poisson$ bracket is a true Poisson bracket if additionally the Jacobi-identity is satisfied, that is,

$$
\{F,\{G, K\}\}+\{G,\{K, F\}\}+\{K,\{F, G\}\}=0 \text { for all } F, G, K: \mathcal{X} \rightarrow \mathbb{R} .
$$

If (and only if) the Jacobi-identity holds, there exist in a neighborhood of every point $x_{0} \in \mathcal{X}$ where $J(x)$ has constant rank local canonical coordinates $(q, p, r)=$ $\left(q_{1}, \ldots, q_{k}, p_{1}, \ldots, p_{k}, r_{1}, \ldots, r_{l}\right)$ for $\mathcal{X}$ in which $J(x)$ takes the form (see e.g., [O])

$$
J(q, p, r)=\left[\begin{array}{ccc}
0 & I_{k} & 0 \\
-I_{k} & 0 & 0 \\
0 & 0 & 0
\end{array}\right],
$$

implying that the Hamiltonian vector field $\dot{x}=J(x) \frac{\partial H}{\partial x}(x)$ takes the form

$$
\begin{aligned}
\dot{q} & =\frac{\partial H}{\partial p}(q, p, r), \\
\dot{p} & =-\frac{\partial H}{\partial q}(q, p, r), \\
\dot{r} & =0
\end{aligned}
$$

which are almost the standard Hamiltonian equations of motion except for the appearance of the conserved quantities $r_{1}, \ldots, r_{l}$. Although in many cases of interest the Jacobi-identity is satisfied, there are clear examples where it is not satisfied (e.g., mechanical systems with nonholonomic kinematic constraints; see [SM1]).

The overall energy-conserving physical system is now obtained by interconnecting the various port-controlled generalized Hamiltonian subsystems as above in a powercontinuous fashion (e.g., by using Kirchhoff's laws). In general this will result in a mixed set of differential and algebraic equations, which nevertheless is expected to be again Hamiltonian in some sense. Indeed, it can be seen that it is an implicit generalized Hamiltonian system, as defined in [SM2, SM3]. The key concept in the definition of an implicit generalized Hamiltonian system is the notion of a generalized Dirac structure, as introduced (in a rather different context) in [C1, D2].

First we concentrate on interconnected energy-conserving physical systems without any remaining external sources; see section 5 for the general case. In this case the Dirac structure for the interconnected system is defined solely on the space of energy-variables. Let $\mathcal{X}$ be an n-dimensional manifold with tangent bundle $T \mathcal{X}$ and cotangent bundle $T^{*} \mathcal{X}$. We define $T \mathcal{X} \oplus T^{*} \mathcal{X}$ as the smooth vector bundle over $\mathcal{X}$ with fiber at each $x \in \mathcal{X}$ given by $T_{x} \mathcal{X} \times T_{x}^{*} \mathcal{X}$. Let $X$ be a smooth vector field and $\alpha$ a smooth one-form on $\mathcal{X}$ respectively. Then we say that the pair $(X, \alpha)$ belongs to a smooth vector subbundle $\mathcal{D} \subset T \mathcal{X} \oplus T^{*} \mathcal{X}($ denoted $(X, \alpha) \in \mathcal{D})$ if $(X(x), \alpha(x)) \in \mathcal{D}(x)$ for every $x \in \mathcal{X}$. Furthermore for a smooth vector subbundle $\mathcal{D} \subset T \mathcal{X} \oplus T^{*} \mathcal{X}$ we define the smooth vector subbundle $D^{\perp} \subset T \mathcal{X} \oplus T^{*} \mathcal{X}$ as

$$
\mathcal{D}^{\perp}=\left\{(X, \alpha) \in T \mathcal{X} \oplus T^{*} \mathcal{X} \mid\langle\alpha \mid \hat{X}\rangle+\langle\hat{\alpha} \mid X\rangle=0, \text { for all }(\hat{X}, \hat{\alpha}) \in \mathcal{D}\right\}
$$


with $\langle\mid\rangle$ denoting the natural pairing between a one-form and a vector field. In (2.8) and throughout in the sequel the pairs $(X, \alpha),(\hat{X}, \hat{\alpha})$ are assumed to be pairs of smooth vector fields and smooth one-forms.

Definition 2.1 (see [C1, D2]). A generalized Dirac structure on an n-dimensional manifold $\mathcal{X}$ is a smooth vector subbundle $\mathcal{D} \subset T \mathcal{X} \oplus T^{*} \mathcal{X}$ such that $\mathcal{D}^{\perp}=\mathcal{D}$.

If $\mathcal{D}$ satisfies an additional closedness (or integrability) condition, then $\mathcal{D}$ defines a Dirac structure; see section 4. Later on we will see that the dimension of the fibers of a generalized Dirac structure on an $n$-dimensional manifold is equal to $n$. By taking $\hat{\alpha}=\alpha, \hat{X}=X$ in $(2.8)$ we obtain

$$
\langle\alpha \mid X\rangle=0 \quad \text { for all }(X, \alpha) \in \mathcal{D} .
$$

Conversely, if $(2.9)$ holds, then for every $(X, \alpha),(\hat{X}, \hat{\alpha}) \in \mathcal{D}$

$$
\begin{aligned}
0=\langle\alpha+\hat{\alpha} \mid X+\hat{X}\rangle & =\langle\alpha \mid X\rangle+\langle\alpha \mid \hat{X}\rangle+\langle\hat{\alpha} \mid X\rangle+\langle\hat{\alpha} \mid \hat{X}\rangle \\
& =\langle\alpha \mid \hat{X}\rangle+\langle\hat{\alpha} \mid X\rangle,
\end{aligned}
$$

and thus $\mathcal{D} \subset \mathcal{D}^{\perp}$. Hence a Dirac structure is a smooth vector subbundle of $T \mathcal{X} \oplus T^{*} \mathcal{X}$ which is maximal with respect to property (2.10) or (2.9).

Let now $\mathcal{X}$ be an $n$-dimensional manifold with a generalized Dirac structure $\mathcal{D}$, and let $H: \mathcal{X} \rightarrow \mathbb{R}$ be a Hamiltonian (energy function). Then the implicit generalized Hamiltonian system on $\mathcal{X}$ corresponding to $\mathcal{D}$ and $H$ is given by the specification (see [SM2])

$$
\left(\dot{x}, \frac{\partial H}{\partial x}(x)\right) \in \mathcal{D}(x)
$$

By (2.9) we immediately obtain the energy conservation property $\frac{d H}{d t}=\left\langle\frac{\partial H}{\partial x}(x) \mid \dot{x}\right\rangle$ $=0$. Note that in general the specification (2.11) puts algebraic constraints on $\mathcal{X}$, since in general there will not exist for every $x \in \mathcal{X}$ a tangent vector $\dot{x} \in T_{x} \mathcal{X}$ such that (2.11) is satisfied. Thus (2.11) is in general a set of differential algebraic equations (DAEs). It can be seen that (2.11) generalizes the notion of an (explicit) generalized Hamiltonian system

$$
\dot{x}=J(x) \frac{\partial H}{\partial x}(x), \quad J(x)=-J^{T}(x),
$$

by noting that

$$
\mathcal{D}=\left\{(X, \alpha) \in T \mathcal{X} \oplus T^{*} \mathcal{X} \mid X(x)=J(x) \alpha(x), x \in \mathcal{X}\right\}
$$

defines a generalized Dirac structure. (If $\alpha^{T}(x) J(x) \hat{\alpha}(x)+\hat{\alpha}^{T}(x) X(x)=0$ for all $\hat{\alpha}$, then $X(x)=J(x) \alpha(x)$.)

A special case of a Dirac structure is that of a constant Dirac structure on a linear space.

Definition 2.2. A constant Dirac structure on a linear $n$-dimensional space $\mathcal{V}$ is a linear subspace $\mathcal{D} \subset \mathcal{V} \times \mathcal{V}^{*}$ with the property that $\mathcal{D}^{\perp}=\mathcal{D}$, where

$$
\mathcal{D}^{\perp}=\left\{\left(v, v^{*}\right) \in \mathcal{V} \times \mathcal{V}^{*} \mid\left\langle v^{*} \mid \hat{v}\right\rangle+\left\langle\hat{v}^{*} \mid v\right\rangle=0 \text { for all }\left(\hat{v}, \hat{v}^{*}\right) \in \mathcal{D}\right\}
$$

where $\langle\mid\rangle$ denotes the natural pairing between $\mathcal{V}$ and $\mathcal{V}^{*}$.

The following proposition is derived straightforwardly. 
Proposition 2.1. Let $\mathcal{V}$ be an $n$-dimensional linear space. A linear subspace $\mathcal{D} \subset \mathcal{V} \times \mathcal{V}^{*}$ defines a constant Dirac structure if and only if $\operatorname{dim} \mathcal{D}=n$ and

$$
\left\langle v^{*} \mid v\right\rangle=0 \quad \text { for all }\left(v, v^{*}\right) \in \mathcal{D} .
$$

Proof. (Sketch; see [SM3] for details.) As in (2.9) and (2.10) we see that if $\mathcal{D}$ defines a constant Dirac structure, then (2.13) holds, while if (2.13) holds, then equivalently

$$
\left\langle v^{*} \mid \hat{v}\right\rangle+\left\langle\hat{v}^{*} \mid v\right\rangle=0 \quad \text { for all }\left(\hat{v}, \hat{v}^{*}\right) \in \mathcal{D} \text {. }
$$

Furthermore, a subspace $\mathcal{D}$ of $\mathcal{V} \times \mathcal{V}^{*}$ defines a Dirac structure if it is maximal with respect to property $(2.14)$, which is equivalent (see [C1]) to the property $\operatorname{dim} \mathcal{D}$ $=n$.

Now let us consider $k$ port-controlled generalized Hamiltonian systems as in (2.1), i.e., for $i=1, \ldots, k$

$$
\begin{aligned}
& \dot{x}_{i}=J_{i}\left(x_{i}\right) \frac{\partial H_{i}}{\partial x_{i}}\left(x_{i}\right)+g_{i}\left(x_{i}\right) f_{i}, \\
& e_{i}=g_{i}^{T}\left(x_{i}\right) \frac{\partial H_{i}}{\partial x_{i}}\left(x_{i}\right), \\
& x_{i} \in \mathcal{X}_{i}, f_{i} \in \mathcal{F}_{i}:=\mathbb{R}^{m_{i}}, e_{i} \in \mathcal{E}_{i}:=\mathcal{F}_{i}^{*}=\mathbb{R}^{m_{i}},
\end{aligned}
$$

with $\mathcal{X}_{i}$ an $n_{i}$-dimensional state space. Consider a general power-conserving interconnection of these systems given by an $\left(m_{1}+\ldots+m_{k}\right)$-dimensional subspace (possibly parametrized by $x_{1}, \ldots, x_{k}$ )

$$
I\left(x_{1}, \ldots, x_{k}\right) \subset \mathcal{F}_{1} \times \cdots \times \mathcal{F}_{k} \times \mathcal{E}_{1} \times \cdots \times \mathcal{E}_{k}
$$

with the property

$$
\left(f_{1}, \ldots, f_{k}, e_{1}, \ldots, e_{k}\right) \in I\left(x_{1}, \ldots, x_{k}\right) \Rightarrow \sum_{i=1}^{k} e_{i}^{T} f_{i}=0 .
$$

Remark 2.1. By Proposition 2.1 it follows that $I\left(x_{1}, \ldots, x_{k}\right)$ defines a constant Dirac structure on $\mathcal{F}_{1} \times \ldots \times \mathcal{F}_{k}$, parameterized by $\left(x_{1}, \ldots, x_{k}\right)$.

Proposition 2.2. Consider $k$ port-controlled generalized Hamiltonian systems (2.15) subject to an interconnection (2.16) satisfying (2.17). Then the resulting interconnected system is an implicit generalized Hamiltonian system with state space $\mathcal{X}:=\mathcal{X}_{1} \times \cdots \times \mathcal{X}_{k}$, Hamiltonian $H\left(x_{1}, \ldots, x_{k}\right):=H_{1}\left(x_{1}\right)+\cdots+H_{k}\left(x_{k}\right)$, and generalized Dirac structure $\mathcal{D}$ on $\mathcal{X}$ given as

$$
\begin{aligned}
& (X, \alpha)=\left(X_{1}, \ldots, X_{k}, \alpha_{1}, \ldots, \alpha_{k}\right) \in \mathcal{D} \Longleftrightarrow \\
& \text { for all } x_{i} \in \mathcal{X}_{i}, \quad i=1, \ldots, k, \quad \exists\left(f_{1}, \ldots, f_{k}, e_{1},\right. \\
& X_{i}\left(x_{i}\right)=J_{i}\left(x_{i}\right) \alpha_{i}\left(x_{i}\right)+g_{i}\left(x_{i}\right) f_{i}, \\
& e_{i}=g_{i}^{T}\left(x_{i}\right) \alpha_{i}\left(x_{i}\right) .
\end{aligned}
$$$$
\text { for all } x_{i} \in \mathcal{X}_{i}, i=1, \ldots, k, \exists\left(f_{1}, \ldots, f_{k}, e_{1}, \ldots, e_{k}\right) \in I\left(x_{1}, \ldots, x_{k}\right) \text { such that }
$$ 
Proof. The main point is in proving that $\mathcal{D}$ given by (2.18) defines a generalized Dirac structure. Let $(X, \alpha)=\left(X_{1}, \ldots, X_{k}, \alpha_{1}, \ldots, \alpha_{k}\right)$ be in $\mathcal{D}^{\perp}$, that is, $\langle\hat{\alpha} \mid X\rangle+$ $\langle\alpha \mid \hat{X}\rangle=0$ for all $(\hat{X}, \hat{\alpha})=\left(\hat{X}_{1}, \ldots, \hat{X}_{k}, \hat{\alpha}_{1}, \ldots, \hat{\alpha}_{k}\right)$ satisfying (2.18). This means

$$
\begin{aligned}
0 & =\sum_{i=1}^{k}\left[\hat{\alpha}_{i}^{T}\left(x_{i}\right) X_{i}\left(x_{i}\right)+\alpha_{i}^{T}\left(x_{i}\right) \hat{X}_{i}\left(x_{i}\right)\right] \\
& =\sum_{i=1}^{k}\left[\hat{\alpha}_{i}^{T}\left(x_{i}\right) X_{i}\left(x_{i}\right)+\alpha_{i}^{T}\left(x_{i}\right) J_{i}\left(x_{i}\right) \hat{\alpha}_{i}\left(x_{i}\right)+\alpha_{i}^{T}\left(x_{i}\right) g_{i}\left(x_{i}\right) \hat{f}_{i}\right] \\
& =\sum_{i=1}^{k}\left(\hat{\alpha}_{i}^{T}\left(x_{i}\right)\left[X_{i}\left(x_{i}\right)-J_{i}\left(x_{i}\right) \alpha_{i}\left(x_{i}\right)\right]+\alpha_{i}^{T}\left(x_{i}\right) g_{i}\left(x_{i}\right) \hat{f}_{i}\right)
\end{aligned}
$$

for all $\hat{\alpha}_{i}, \hat{f}_{i}$ such that $\hat{e}_{i}=g_{i}^{T}\left(x_{i}\right) \hat{\alpha}_{i}\left(x_{i}\right)$ satisfies $\left(\hat{f}_{1}, \ldots, \hat{f}_{k}, \hat{e}_{1}, \ldots, \hat{e}_{k}\right) \in I\left(x_{1}\right.$, $\left.\ldots, x_{k}\right)$. Letting first $\hat{f}_{i}=0$ and $\hat{e}_{i}=0$, we obtain

$$
\sum_{i=1}^{k} \hat{\alpha}_{i}^{T}\left(x_{i}\right)\left[X_{i}\left(x_{i}\right)-J_{i}\left(x_{i}\right) \alpha_{i}\left(x_{i}\right)\right]=0
$$

for all $\hat{\alpha}_{i}\left(x_{i}\right)$ such that $g_{i}^{T}\left(x_{i}\right) \hat{\alpha}_{i}\left(x_{i}\right)=0$. This means that there exist vectors $f_{1}, \ldots, f_{k}$ such that

$$
X_{i}\left(x_{i}\right)=J_{i}\left(x_{i}\right) \alpha_{i}\left(x_{i}\right)+g_{i}\left(x_{i}\right) f_{i} .
$$

Substitution into (2.19) yields

$$
\begin{aligned}
0 & =\sum_{i=1}^{k}\left(\hat{\alpha}_{i}^{T}\left(x_{i}\right) g_{i}\left(x_{i}\right) f_{i}+\alpha_{i}^{T}\left(x_{i}\right) g_{i}\left(x_{i}\right) \hat{f}_{i}\right) \\
& =\sum_{i=1}^{k}\left(\hat{e}_{i}^{T} f_{i}+e_{i}^{T} \hat{f}_{i}\right)
\end{aligned}
$$

for all $\hat{f}_{i}$ and $\hat{e}_{i}=g_{i}^{T}\left(x_{i}\right) \hat{\alpha}_{i}\left(x_{i}\right)$ satisfying $\left(\hat{f}_{1}, \ldots, \hat{f}_{k}, \hat{e}_{1}, \ldots, \hat{e}_{k}\right) \in I\left(x_{1}, \ldots, x_{k}\right)$. If $g_{i}^{T}\left(x_{i}\right)$ is surjective for all $i=1, \ldots, k$, this means that $(2.22)$ is satisfied for all $\left(\hat{f}_{1}, \ldots, \hat{f}_{k}, \hat{e}_{1}, \ldots, \hat{e}_{k}\right) \in I\left(x_{1}, \ldots, x_{k}\right)$, and by Proposition 2.1 and Remark 2.1 this implies that $\left(f_{1}, \ldots, f_{k}, e_{1}, \ldots, e_{k}\right) \in I\left(x_{1}, \ldots, x_{k}\right)$ and thus $(X, \alpha) \in \mathcal{D}$. In general we proceed as follows. Define the space of achievable flows and efforts

$$
C\left(x_{1}, \ldots, x_{k}\right):=\left\{\left(\hat{f}_{1}, \ldots, \hat{f}_{k}, \hat{e}_{1}, \ldots, \hat{e}_{k}\right) \mid \hat{f}_{i} \in \mathcal{F}_{i}, \hat{e}_{i} \in \operatorname{Im} g_{i}^{T}\left(x_{i}\right), i=1, \ldots, k\right\} .
$$

Then (2.22) implies that

$$
\begin{aligned}
\left(f_{1}, \ldots, f_{k}, e_{1}, \ldots, e_{k}\right) & \in\left(I\left(x_{1}, \ldots, x_{k}\right) \cap C\left(x_{1}, \ldots, x_{k}\right)\right)^{\perp} \\
& =I^{\perp}\left(x_{1}, \ldots, x_{k}\right)+C^{\perp}\left(x_{1}, \ldots, x_{k}\right)
\end{aligned}
$$

where $\perp$ denotes orthogonal complement with respect to property (2.22). By Proposition 2.1 it follows that $I^{\perp}\left(x_{1}, \ldots, x_{k}\right)=I\left(x_{1}, \ldots, x_{k}\right)$, while $C^{\perp}\left(x_{1}, \ldots, x_{k}\right)$ is seen to be given as

$$
C^{\perp}\left(x_{1}, \ldots, x_{k}\right)=\left\{\left(f_{1}, \ldots, f_{k}, e_{1}, \ldots, e_{k}\right) \mid f_{i} \in \operatorname{ker} g_{i}\left(x_{i}\right), e_{i}=0, i=1, \ldots, k\right\} .
$$


Thus there exist flow vectors $f_{1}^{\prime}, \ldots, f_{k}^{\prime}$ such that $\left(f_{1}^{\prime}, \ldots, f_{k}^{\prime}, e_{1}, \ldots, e_{k}\right) \in I\left(x_{1}\right.$, $\left.\ldots, x_{k}\right)$, with $X_{i}\left(x_{i}\right)=J_{i}\left(x_{i}\right) \alpha_{i}\left(x_{i}\right)+g_{i}\left(x_{i}\right) f_{i}^{\prime}, e_{i}=g_{i}^{T}\left(x_{i}\right) \alpha_{i}\left(x_{i}\right)$, showing that $(X, \alpha) \in \mathcal{D}$. Hence $\mathcal{D}^{\perp} \subset \mathcal{D}$. Since it is easily seen that $\mathcal{D} \subset \mathcal{D}^{\perp}$, this shows that $\mathcal{D}$ defines a Dirac structure.

We note that the definition of a power-conserving interconnection is very general and for example includes Kirchhoff's laws for electrical systems, the interconnection relations for generalized velocities and forces for interconnected mechanical systems (Newton's third law), as well as transformers in electrical circuits and kinematic pairs in multibody systems.

From a classical control point of view an important example of a power-conserving interconnection is the standard feedback interconnection.

EXAMPle 2.1. Consider two input-state-output systems ("plant" and "controller")

$$
\begin{aligned}
& \dot{x}_{i}=g_{i}\left(x_{i}, u_{i}\right), \\
& y_{i}=h_{i}\left(x_{i}\right), \quad u_{i}, y_{i} \in \mathbb{R}^{m}, \quad i=1,2,
\end{aligned}
$$

and impose the (negative) feedback interconnection

$$
\begin{aligned}
& u_{2}=y_{1}, \\
& u_{1}=-y_{2},
\end{aligned}
$$

leading to the explicit system

$$
\begin{aligned}
& \dot{x}_{1}=g_{1}\left(x_{1},-h_{2}\left(x_{2}\right)\right), \\
& \dot{x}_{2}=g_{2}\left(x_{2}, h_{1}\left(x_{1}\right)\right) .
\end{aligned}
$$

If we equate the input vectors $u_{i}$ with flow vectors, and the output vectors $y_{i}$ with effort vectors, then (2.24) is a power-conserving interconnection. Proposition 2.2 applied to this particular case says that if both systems in (2.23) are Hamiltonian, then also (2.25) is Hamiltonian. This can be regarded as a special instance of the passivity theorem in input-output stability theory.

3. Representations of generalized Dirac structures and implicit generalized Hamiltonian systems. There are different ways of representing generalized Dirac structures, and consequently of writing the equations of an implicit generalized Hamiltonian system. These representations each have their own advantages and are connected to different but equivalent ways of mathematically modelling the energyconserving physical systems.

Before going into these representations we first note that a generalized Dirac structure $\mathcal{D}$ on an $n$-dimensional manifold $\mathcal{X}$ defines the smooth distributions

$$
\begin{aligned}
& G_{0}=\{X \in T \mathcal{X} \mid(X, 0) \in \mathcal{D}\}, \\
& G_{1}=\left\{X \in T \mathcal{X} \mid \exists \alpha \in T^{*} \mathcal{X} \text { s.t. }(X, \alpha) \in \mathcal{D}\right\}
\end{aligned}
$$

and the smooth codistributions

$$
\begin{aligned}
& P_{0}=\left\{\alpha \in T^{*} \mathcal{X} \mid(0, \alpha) \in \mathcal{D}\right\}, \\
& P_{1}=\left\{\alpha \in T^{*} \mathcal{X} \mid \exists X \in T \mathcal{X} \text { s.t. }(X, \alpha) \in \mathcal{D}\right\} .
\end{aligned}
$$

Define for any smooth distribution $G$ the smooth codistribution ann $G$ as

$$
\text { ann } G=\left\{\alpha \in T^{*} \mathcal{X} \mid\langle\alpha \mid X\rangle=0 \text { for all } X \in G\right\}
$$


and for any smooth codistribution $P$ the smooth distribution ker $P$ as

$$
\text { ker } P=\{X \in T \mathcal{X} \mid\langle\alpha \mid X\rangle=0 \text { for all } \alpha \in P\} .
$$

The smooth (co)distributions $G_{0}, G_{1}$ and $P_{0}, P_{1}$ are related as follows.

Proposition 3.1. Let $\mathcal{D}$ be a generalized Dirac structure on $\mathcal{X}$ and define $G_{0}$, $G_{1}, P_{0}, P_{1}$ as in (3.1), (3.2). Then

1. $G_{0}=\operatorname{ker} P_{1}, P_{0}=\operatorname{ann} G_{1}$;

2. $P_{1} \subset$ ann $G_{0}, G_{1} \subset$ ker $P_{0}$, with equality if $G_{1}$, respectively, $P_{1}$, is constantdimensional.

Proof.

1. $Z \in G_{0}$ if and only if $(Z, 0) \in \mathcal{D}$, if and only if

$$
\langle 0 \mid X\rangle+\langle\alpha \mid Z\rangle=0 \text { for all }(X, \alpha) \in \mathcal{D}
$$

or equivalently $\langle\alpha \mid Z\rangle=0$ for all $\alpha \in P_{1}$. Thus $G_{0}=$ ker $P_{1}$. Similarly $\beta \in P_{0}$ if and only if $(0, \beta) \in \mathcal{D}$, if and only if $\langle\beta \mid X\rangle=0$ for all $X \in G_{1}$, which implies $P_{0}=\operatorname{ann} G_{1}$.

2. This follows from property 1 and the inequalities $P \subset$ ann ker $P, G \subset$ ker ann $G$, for any smooth (co)distribution $P$ and $G$, with equality if $P$ and $G$ are constant-dimensional [NS].

REMARK 3.1. The distribution $G_{1}$ and the co-distribution $P_{1}$ have the following interpretation. Consider the implicit generalized Hamiltonian system (2.11) corresponding to a generalized Dirac structure $\mathcal{D}$ and a Hamiltonian $H$. Then the distribution $G_{1}$ describes the set of admissible flows $\dot{x}$. In particular, if $G_{1}$ is constantdimensional and involutive then there are $\left(n-\operatorname{dim} G_{1}\right)$ independent conserved quantities for (2.11). Dually the codistribution $P_{1}$ describes the set of algebraic constraints of (2.11), i.e.,

$$
\frac{\partial H}{\partial x}(x) \in P_{1}(x)
$$

Definition 3.1. A point $x \in \mathcal{X}$ is a regular point for the Dirac structure $\mathcal{D}$ on $\mathcal{X}$ if the dimension of $G_{1}$ and $P_{1}$ (and hence, see Proposition 3.1, of $G_{0}, P_{0}$ ) is constant in a neighborhood of $x$.

At every regular point $x \in X$ we have

$$
\mathcal{D}^{\perp}(x)=\left\{\left(v, v^{*}\right) \in T_{x} \mathcal{X} \times T_{x}^{*} \mathcal{X} \mid\left\langle v^{*} \mid \hat{v}\right\rangle+\left\langle\hat{v}^{*} \mid v\right\rangle=0 \text { for all }\left(\hat{v}, \hat{v}^{*}\right) \in \mathcal{D}(x)\right\}
$$

and since $\mathcal{D}^{\perp}(x)=\mathcal{D}(x)$, we may regard $\mathcal{D}(x) \subset T_{x} \mathcal{X} \times T_{x}^{*} \mathcal{X}$ as a constant Dirac structure on $T_{x} \mathcal{X}$ (see Definition 2.2). Invoking Proposition 2.1 we deduce that $\operatorname{dim} \mathcal{D}(x)=n$ for every regular point $x \in \mathcal{X}$. Since the set of regular points is open and dense in $\mathcal{X}$, and $\mathcal{D}$ is a vector subbundle, it thus follows that

$$
\operatorname{dim} \mathcal{D}(x)=n \text { for all } x \in \mathcal{X},
$$

and therefore we may regard $\mathcal{D}(x) \subset T_{x} \mathcal{X} \times T_{x}^{*} \mathcal{X}$ as a constant Dirac structure on $T_{x} \mathcal{X}$ for every $x \in \mathcal{X}$. In particular it follows, since $\mathcal{D}$ is a smooth vector subbundle, that locally about every point in $\mathcal{X}$ we may find $n \times n$ matrices $E(x)$ and $F(x)$, depending smoothly on $x$, such that locally

$$
\begin{aligned}
& \mathcal{D}(x)=\left\{\left(v, v^{*}\right) \in T_{x} \mathcal{X} \times T_{x}^{*} \mathcal{X} \mid F(x) v=E(x) v^{*}\right\}, \\
& \operatorname{rank}[F(x):-E(x)]=n .
\end{aligned}
$$


Furthermore, because $\mathcal{D}=\mathcal{D}^{\perp}$ necessarily (see [SM2])

$$
E(x) F^{T}(x)+F(x) E^{T}(x)=0 .
$$

We will refer to this local representation (3.8), (3.9) of a Dirac structure as representation I. Given a Hamiltonian $H: \mathcal{X} \rightarrow \mathbb{R}$ the corresponding implicit generalized Hamiltonian system in representation $\mathrm{I}$ is locally given as

$$
F(x) \dot{x}=E(x) \frac{\partial H}{\partial x}(x) .
$$

EXAMPLE 3.1 ([SM2]; see also [MSB2]). An LC-circuit is composed of a set of (multiport) inductors and capacitors interconnected through their ports by the network graph. An n-port inductor is defined by flux linkage variables $\phi \in \mathbb{R}^{n}$ (the energy variables) and an energy function $H_{L}(\phi)$. The port variables are the voltages $v_{L} \in \mathbb{R}^{n}$ and the currents $i_{L} \in \mathbb{R}^{n}$ defined as

$$
v_{L}=\dot{\phi}, \quad i_{L}=\frac{\partial H_{L}}{\partial \phi} .
$$

Similarly, an n-port capacitor is defined by charge variables $q \in \mathbb{R}^{n}$ and energy function $H_{C}(q)$, with port variables the currents $i_{C} \in \mathbb{R}^{n}$ and voltages $v_{C} \in \mathbb{R}^{n}$ defined as

$$
i_{C}=\dot{q}, \quad v_{C}=\frac{\partial H_{C}}{\partial q} .
$$

By Kirchhoff's laws we obtain $n_{L}+n_{C}$ independent equations

$$
F_{C} i_{C}+E_{C} i_{L}=0, \quad F_{L} v_{L}+E_{L} v_{C}=0
$$

for certain matrices $F_{C}, F_{L}, E_{C}$, and $E_{L}$ satisfying (Tellegen's theorem)

$$
E_{C} F_{L}^{T}+F_{C} E_{L}^{T}=0 .
$$

Using (3.11), (3.12) and defining the total energy $H(q, \phi)=H_{L}(\phi)+H_{C}(q)$, we may rewrite (3.13) as the implicit generalized Hamiltonian system

$$
\underbrace{\left[\begin{array}{cc}
F_{C} & 0 \\
0 & F_{L}
\end{array}\right]}_{F}\left[\begin{array}{c}
\dot{q} \\
\dot{\phi}
\end{array}\right]=\underbrace{\left[\begin{array}{cc}
0 & -E_{C} \\
-E_{L} & 0
\end{array}\right]}_{E}\left[\begin{array}{c}
\frac{\partial H}{\partial q} \\
\frac{\partial H}{\partial \phi}
\end{array}\right],
$$

where $E F^{T}+F E^{T}=0$ by (3.14).

Two other useful types of representations of generalized Dirac structures, which admit a global and coordinate-free definition, can be given provided an extra regularity condition is satisfied. We will denote them as representation II and representation III, respectively.

TheOREM 3.1 (representation II). Let $\mathcal{X}$ be an $n$-dimensional manifold. Let $G$ be a constant-dimensional distribution on $\mathcal{X}$, and $J(x): T_{x}^{*} \mathcal{X} \rightarrow T_{x} \mathcal{X}, x \in \mathcal{X}, a$ skew-symmetric vector bundle map. Then

$$
\mathcal{D}=\left\{(X, \alpha) \in T \mathcal{X} \oplus T^{*} \mathcal{X} \mid X(x)-J(x) \alpha(x) \in G(x), x \in \mathcal{X}, \alpha \in \text { ann } G\right\}
$$

defines a generalized Dirac structure. Conversely, let $\mathcal{D}$ be any generalized Dirac structure having the property that the codistribution $P_{1}$ (see (3.2)) is constant-dimensional. 
Then there exists a skew-symmetric vector bundle map $J(x): P_{1}(x) \rightarrow\left(P_{1}(x)\right)^{*}$, $x \in \mathcal{X}$, which locally can be extended to a skew-symmetric vector bundle map $J(x)$ : $T_{x}^{*} \mathcal{X} \rightarrow T_{x} \mathcal{X}, x \in \mathcal{X}$, such that $\mathcal{D}$ is given by (3.16) with $G:=\operatorname{ker} P_{1}$.

Proof (see also [C1] for the constant case). Let $\mathcal{D}$ be given by (3.16). We have to show that $\mathcal{D}^{\perp}=\mathcal{D}$.

1. Take $(X, \alpha)=(J \alpha+Z, \alpha) \in \mathcal{D}$, with $Z \in G$. Then for all $(\hat{X}, \hat{\alpha})=(J \hat{\alpha}+$ $\hat{Z}, \hat{\alpha}) \in \mathcal{D}, \hat{Z} \in G$

$$
\langle\alpha \mid \hat{X}\rangle+\langle\hat{\alpha} \mid X\rangle=\langle\alpha \mid J \hat{\alpha}\rangle+\langle\hat{\alpha} \mid J \alpha\rangle+\langle\alpha \mid \hat{Z}\rangle+\langle\hat{\alpha} \mid Z\rangle=0
$$

because $J(x)$ is skew-symmetric, and $\alpha, \hat{\alpha} \in \operatorname{ann} G$.

2. Take $(X, \alpha) \in \mathcal{D}^{\perp}$, that is for all $(\hat{X}, \hat{\alpha})=(J \hat{\alpha}+\hat{Z}, \hat{\alpha}) \in \mathcal{D}, \hat{Z} \in G, \hat{\alpha} \in \operatorname{ann} G$

$$
0=\langle\alpha \mid \hat{X}\rangle+\langle\hat{\alpha} \mid X\rangle=\langle\alpha \mid J \hat{\alpha}\rangle+\langle\alpha \mid \hat{Z}\rangle+\langle\hat{\alpha} \mid X\rangle
$$

First let $\hat{Z}=0$. Then

$$
0=\langle\alpha \mid J \hat{\alpha}\rangle+\langle\hat{\alpha} \mid X\rangle=\langle\hat{\alpha} \mid X-J \alpha\rangle
$$

for all $\hat{\alpha} \in \operatorname{ann} G$, implying that $X-J \alpha \in \operatorname{ker}$ ann $G=G$, since $G$ is constantdimensional. Now let $\hat{\alpha}=0$. Then

$$
0=\langle\alpha \mid \hat{Z}\rangle
$$

for all $\hat{Z} \in G$, implying that $\alpha \in$ ann $G$.

Conversely, let $\mathcal{D}$ be a generalized Dirac structure on $\mathcal{X}$, with $P_{1}$ constant-dimensional. Then we define for every $x \in \mathcal{X}$ a linear map

$$
J(x): P_{1}(x) \subset T_{x}^{*} \mathcal{X} \rightarrow\left(P_{1}(x)\right)^{*} \subset T_{x} \mathcal{X}
$$

as follows. Let $v^{*} \in P_{1}(x)$, that is, there exists $v \in T_{x} \mathcal{X}$ such that $\left(v, v^{*}\right) \in \mathcal{D}(x)$. Then define

$$
J(x) v^{*}=v \in\left(P_{1}(x)\right)^{*} .
$$

To see that $J(x)$ is well-defined, let also $\left(\hat{v}, v^{*}\right) \in \mathcal{D}(x)$. Then $(v-\hat{v}, 0) \in \mathcal{D}(x)$, which means $v-\hat{v} \in G_{0}(x)=\operatorname{ker} P_{1}(x)$, and thus $v$ and $\hat{v}$ define the same linear function on $P_{1}(x)$. Skew-symmetry of the map $J(x): P_{1}(x) \rightarrow\left(P_{1}(x)\right)^{*}$ follows from

$$
\left\langle\hat{v}^{*} \mid v\right\rangle+\left\langle v^{*} \mid \hat{v}\right\rangle=0 .
$$

for all $\left(v, v^{*}\right),\left(\hat{v}, \hat{v}^{*}\right) \in \mathcal{D}(x)$. Finally we may locally extend $J(x)$ to a skew-symmetric map from $T_{x} \mathcal{X}$ to $T_{x}^{*} \mathcal{X}$. Now, let $\left(v, v^{*}\right) \in \mathcal{D}(x)$. Then by (3.17) $v=J(x) v^{*}$ modulo $G(x):=\operatorname{ker} P_{1}(x)$, while $v^{*} \in P_{1}(x)$, and thus $\mathcal{D}$ is indeed given by (3.16).

REMARK 3.2. Note (see (3.17)) that the kernel of $J(x): P_{1}(x) \rightarrow\left(P_{1}(x)\right)^{*}$ is given by $P_{0}(x)$.

Given a Hamiltonian $H: \mathcal{X} \rightarrow \mathbb{R}$ the equations of the implicit generalized Hamiltonian system corresponding to representation II now take the form

$$
\begin{aligned}
\dot{x} & =J(x) \frac{\partial H}{\partial x}(x)+g(x) \lambda, \\
0 & =g^{T}(x) \frac{\partial H}{\partial x}(x),
\end{aligned}
$$


where $g(x)$ is any full rank matrix such that $\operatorname{Im} g(x)=G(x)$. The variables $\lambda$ can be seen as Lagrange multipliers, required to keep the constraint equations $g^{T}(x) \frac{\partial H}{\partial x}(x)=$ 0 to be satisfied for all time. Note that (3.18) can be also interpreted as a portcontrolled generalized Hamiltonian system (see section 2) with the efforts (or outputs) $e$ set equal to zero.

"Dualizing" representation II we obtain the following.

Theorem 3.2 (representation III). Let $\mathcal{X}$ be an n-dimensional manifold. Let $P$ be a constant-dimensional codistribution on $\mathcal{X}$, and $\omega(x): T_{x} \mathcal{X} \rightarrow T_{x}^{*} \mathcal{X}, x \in \mathcal{X}, a$ skew-symmetric vector bundle map. Then

$$
\mathcal{D}=\left\{(X, \alpha) \in T \mathcal{X} \oplus T^{*} \mathcal{X} \mid \alpha(x)-\omega(x) X(x) \in P(x), x \in \mathcal{X}, X \in \text { ker } P\right\}
$$

defines a generalized Dirac structure. Conversely, let $\mathcal{D}$ be any generalized Dirac structure having the property that the distribution $G_{1}$ (see (3.1)) is constant-dimensional. Then there exists a skew-symmetric vector bundle map $\omega(x): G_{1}(x) \rightarrow\left(G_{1}(x)\right)^{*}$, $x \in \mathcal{X}$, which locally can be extended to a skew-symmetric vector bundle map $\omega(x)$ : $T_{x} \mathcal{X} \rightarrow T_{x}^{*} \mathcal{X}, x \in \mathcal{X}$, such that $\mathcal{D}$ is given by (3.19) with $P:=$ ann $G_{1}$.

Proof. The proof is completely dual to the proof of Theorem 3.1

REMARK 3.3 (see Remark 3.2). The kernel of $\omega(x): G_{1}(x) \rightarrow\left(G_{1}(x)\right)^{*}$ is given by $G_{0}(x)$.

The equations of an implicit generalized Hamiltonian system corresponding to Representation III and a Hamiltonian $H$ take the form

$$
\begin{aligned}
\frac{\partial H}{\partial x}(x) & =\omega(x) \dot{x}+p^{T}(x) \lambda, \\
0 & =p(x) \dot{x},
\end{aligned}
$$

where $p(x)$ is any full rank matrix such that $\operatorname{Im} p(x)=P(x)$. A main feature of $(3.20)$ in comparison with (3.18) is that in (3.20) the flow constraints $p^{T}(x) \dot{x}=0$ are made explicit, while in (3.18) the algebraic constraints $g^{T}(x) \frac{\partial H}{\partial x}(x)=0$ are distinguished.

EXAMPLE 3.2. Let $Q$ be an n-dimensional configuration manifold of a mechanical system. Classical (kinematic) constraints are given in local coordinates $q=$ $\left(q_{1}, \ldots, q_{n}\right)$ for $Q$ as

$$
A^{T}(q) \dot{q}=0
$$

with $A(q)$ an $n \times k$ matrix, $k \leq n$, with entries depending smoothly on $q$. We will assume that $A(q)$ has rank equal to $k$ everywhere. The constrained Hamiltonian equations on $T^{*} Q$ are classically given as (see, e.g., [SM1])

$$
\begin{aligned}
{\left[\begin{array}{c}
\dot{q} \\
\dot{p}
\end{array}\right] } & =\underbrace{\left[\begin{array}{cc}
0 & I_{n} \\
-I_{n} & 0
\end{array}\right]}_{J}\left[\begin{array}{c}
\frac{\partial H}{\partial q}(q, p) \\
\frac{\partial H}{\partial p}(q, p)
\end{array}\right]+\left[\begin{array}{c}
0 \\
A(q)
\end{array}\right] \lambda, \\
0 & =\left[\begin{array}{ll}
0 & A^{T}(q)
\end{array}\right]\left[\begin{array}{c}
\frac{\partial H}{\partial q}(q, p) \\
\frac{\partial H}{\partial p}(q, p)
\end{array}\right] .
\end{aligned}
$$

Here the constraint forces $A(q) \lambda$, with $\lambda \in \mathbb{R}^{k}$, are uniquely determined by the requirement that the constraints (3.21) have to be satisfied for all time. It is straightforward 
to see that an equivalent description of the equations (3.22) is given as follows

$$
\begin{aligned}
{\left[\begin{array}{c}
\frac{\partial H}{\partial q}(q, p) \\
\frac{\partial H}{\partial p}(q, p)
\end{array}\right] } & =\underbrace{\left[\begin{array}{cc}
0 & -I_{n} \\
I_{n} & 0
\end{array}\right]}_{\omega}\left[\begin{array}{c}
\dot{q} \\
\dot{p}
\end{array}\right]+\left[\begin{array}{c}
A(q) \\
0
\end{array}\right] \lambda, \\
0 & =\left[\begin{array}{ll}
A^{T}(q) & 0
\end{array}\right]\left[\begin{array}{c}
\dot{q} \\
\dot{p}
\end{array}\right] .
\end{aligned}
$$

Let $G$ and $P$ be the distribution and the codistribution, respectively, on $T^{*} Q$ spanned by the columns of the matrix $\left[\begin{array}{c}0(q)\end{array}\right]$ and the rows of the matrix $\left[\begin{array}{ll}A^{T}(q) & 0\end{array}\right]$, respectively. Then, since both $J$ and $\omega$ are skew-symmetric, it follows from Theorems 3.1 and 3.2 that the pairs $(J, G)$ and $(\omega, P)$ define representation II and representation III, respectively, of the same generalized Dirac structure. We will refer to this generalized Dirac structure as $\mathcal{D}_{A}$.

As the last part of this section we will now briefly show how we can directly go from representation I to a local version of representation II or III, and vice versa. This is particularly useful in analysis, where some aspects may be more easily studied in one representation, while others are easier to address in a different representation. The transformation from representation II or III to I is direct and consists of eliminating the Lagrange multipliers $\lambda$. Indeed, consider the implicit generalized Hamiltonian system (3.18) corresponding to representation II. Since rank $g(x)=k$ for all $x \in \mathcal{X}$, we can locally find an $(n-k) \times n$ matrix $s(x)$ of constant rank $n-k$ such that $s(x) g(x)=0$. Premultiplying the first $n$ equations of (3.18) by $s(x)$ then transforms (3.18) into the following $n$ equations:

$$
\left[\begin{array}{c}
s(x) \\
0
\end{array}\right] \dot{x}=\left[\begin{array}{c}
s(x) J(x) \\
g^{T}(x)
\end{array}\right] \frac{\partial H}{\partial x}(x),
$$

which is easily seen to be of the form (3.10) with $F(x)=\left[\begin{array}{c}s(x) \\ 0\end{array}\right]$ and $E(x)=$ $\left[\begin{array}{c}s(x) J(x) \\ g^{T}(x)\end{array}\right]$ satisfying $(3.8),(3.9)$. The transformation from Representation III to I is completely similar.

EXAMPLE 3.3. Consider again the mechanical system with kinematic constraints in Example 3.2. Since $\operatorname{rank} A(q)=k$ for all $q \in Q$, we can locally find an $(n-k) \times n$ matrix $S(q)$ of constant rank $n-k$ such that $S(q) A(q)=0$. Premultiplying the first $2 n$ equations of $(3.22)$ by the $(2 n-k) \times 2 n$ matrix

$$
\left[\begin{array}{cc}
I_{n} & 0 \\
0 & S(q)
\end{array}\right]
$$

of constant rank $2 n-k$ then transforms (3.22) into the following $2 n$ equations:

$$
\left[\begin{array}{cc}
I_{n} & 0 \\
0 & S(q) \\
0 & 0
\end{array}\right]\left[\begin{array}{c}
\dot{q} \\
\dot{p}
\end{array}\right]=\left[\begin{array}{cc}
0 & I_{n} \\
-S(q) & 0 \\
0 & A^{T}(q)
\end{array}\right]\left[\begin{array}{l}
\frac{\partial H}{\partial q}(q, p) \\
\frac{\partial H}{\partial p}(q, p)
\end{array}\right] .
$$

The transformation from representation I to II or III is more substantial. Consider representation I as given by (3.8), (3.9). Since

$$
\operatorname{ker}[F(x):-E(x)]=\operatorname{Im}\left[\begin{array}{c}
E^{T}(x) \\
-F^{T}(x)
\end{array}\right]
$$


we deduce that locally

$$
G_{1}(x)=\operatorname{Im} E^{T}(x), \quad P_{1}(x)=\operatorname{Im} F^{T}(x)
$$

(while $G_{0}(x)=\operatorname{ker} F(x), P_{0}(x)=\operatorname{ker} E(x)$ if $F(x)$ (respectively, $E(x)$ ) has constant rank). In order to obtain representation II we need to assume that $P_{1}$ has constant dimension (see Theorem 3.1), or equivalently by (3.28), $F(x)$ has constant rank. Then we may always locally transform the equations $F(x) v=E(x) v^{*}$ into the form

$$
\left[\begin{array}{c}
F_{1}(x) \\
0
\end{array}\right] v=\left[\begin{array}{c}
E_{1}(x) \\
E_{2}(x)
\end{array}\right] v^{*}
$$

where $F_{1}(x)$ has full row rank for every $x$ in this neighborhood. Since

$$
0=E(x) F^{T}(x)+F(x) E^{T}(x)=\left[\begin{array}{cc}
E_{1}(x) F_{1}^{T}(x)+F_{1}(x) E_{1}^{T}(x) & F_{1}(x) E_{2}^{T}(x) \\
E_{2}(x) F_{1}^{T}(x) & 0
\end{array}\right]
$$

it follows that

$$
E_{1}(x) F_{1}^{T}(x)+F_{1}(x) E_{1}^{T}(x)=0
$$

and $E_{2}^{T}(x) F_{1}(x)=0$, or actually since $\operatorname{rank}[F(x):-E(x)]=n$

$$
\operatorname{ker} F_{1}(x)=\operatorname{Im} E_{2}^{T}(x) .
$$

By injectivity of $F_{1}^{T}(x)$ it follows that there exists an $n \times n$ matrix $J(x)$ satisfying $J(x) F_{1}^{T}(x)=-E_{1}^{T}(x)$, which is by (3.31) skew-symmetric on $\operatorname{Im} F_{1}^{T}(x)$, and extendable to a skew-symmetric matrix on $\mathbb{R}^{n}$. Thus the equations (3.29) can be written as

$$
\begin{aligned}
& v-J(x) v^{*} \in \operatorname{ker} F_{1}(x)=\operatorname{Im} E_{2}^{T}(x), \\
& 0=E_{2}(x) v^{*}
\end{aligned}
$$

or equivalently, defining the constant rank matrix $g(x):=E_{2}^{T}(x)$,

$$
\begin{aligned}
& v=J(x) v^{*}+g(x) \lambda, \\
& 0=g^{T}(x) v^{*}
\end{aligned}
$$

which is representation II. Representation III can be obtained similarly by manipulating instead of $F(x)$ the constant rank matrix $E(x)$.

4. Closedness of generalized Dirac structures. The Dirac structures $\mathcal{D}$ of Definition 2.1 are called generalized because they do not necessarily satisfy the following closedness (or integrability) condition.

Definition 4.1 (see [D2]). A generalized Dirac structure $\mathcal{D}$ on $\mathcal{X}$ is called closed (or simply a Dirac structure) if for arbitrary $\left(X_{1}, \alpha_{1}\right),\left(X_{2}, \alpha_{2}\right)$, and $\left(X_{3}, \alpha_{3}\right) \in \mathcal{D}$ there holds

$$
\left\langle L_{X_{1}} \alpha_{2} \mid X_{3}\right\rangle+\left\langle L_{X_{2}} \alpha_{3} \mid X_{1}\right\rangle+\left\langle L_{X_{3}} \alpha_{1} \mid X_{2}\right\rangle=0 .
$$

The following theorem gives a very useful characterization of closedness of a generalized Dirac structure.

Theorem 4.1 (cf. [D2, Theorem 2.1]; see also [C1]). $\mathcal{D}$ is closed if and only if

$$
\left(\left[X_{1}, X_{2}\right], \mathbf{i}_{X_{1}} \mathbf{d} \alpha_{2}-\mathbf{i}_{X_{2}} \mathbf{d} \alpha_{1}+\mathbf{d}\left\langle\alpha_{2} \mid X_{1}\right\rangle\right) \in \mathcal{D} \text { for all }\left(X_{1}, \alpha_{1}\right),\left(X_{2}, \alpha_{2}\right) \in \mathcal{D} \text {. }
$$


Proof. First note that the identities (see, e.g., $[\mathrm{AMR}]$ )

$$
\begin{gathered}
L_{X} \alpha=\mathbf{d i}_{X} \alpha+\mathbf{i}_{X} \mathbf{d} \alpha, \\
\mathbf{i}_{[X, Y]} \alpha=L_{X} \mathbf{i}_{Y} \alpha-\mathbf{i}_{Y} L_{X} \alpha
\end{gathered}
$$

are satisfied for all vector fields $X, Y$ and $k$-forms $\alpha$ on $\mathcal{X}$. (The formula (4.3) is also known as Cartan's magic formula.) Hence,

$$
\begin{aligned}
\left\langle L_{X} \alpha \mid Y\right\rangle & =\langle\mathbf{d}\langle\alpha \mid X\rangle \mid Y\rangle+\mathbf{d} \alpha(X, Y), \\
\langle\alpha \mid[X, Y]\rangle & =-\langle\mathbf{d}\langle\alpha \mid X\rangle \mid Y\rangle+\left\langle L_{Y} \alpha \mid X\right\rangle
\end{aligned}
$$

for all vector fields $X, Y$ and one-forms $\alpha$ on $\mathcal{X}$.

Now take arbitrary $\left(X_{1}, \alpha_{1}\right),\left(X_{2}, \alpha_{2}\right),\left(X_{3}, \alpha_{3}\right) \in \mathcal{D}$. Then

$$
\begin{aligned}
& \left\langle\mathbf{i}_{X_{1}} \mathbf{d} \alpha_{2}-\mathbf{i}_{X_{2}} \mathbf{d} \alpha_{1}+\mathbf{d}\left\langle\alpha_{2} \mid X_{1}\right\rangle \mid X_{3}\right\rangle+\left\langle\alpha_{3} \mid\left[X_{1}, X_{2}\right]\right\rangle \\
& \quad=\left\langle\mathbf{i}_{X_{1}} \mathbf{d} \alpha_{2} \mid X_{3}\right\rangle-\left\langle\mathbf{i}_{X_{2}} \mathbf{d} \alpha_{1} \mid X_{3}\right\rangle+\left\langle\mathbf{d}\left\langle\alpha_{2} \mid X_{1}\right\rangle \mid X_{3}\right\rangle+\left\langle\alpha_{3} \mid\left[X_{1}, X_{2}\right]\right\rangle \\
& \quad=\mathbf{d} \alpha_{2}\left(X_{1}, X_{3}\right)+\left\langle\mathbf{d}\left\langle\alpha_{2} \mid X_{1}\right\rangle \mid X_{3}\right\rangle-\mathbf{d} \alpha_{1}\left(X_{2}, X_{3}\right)+\left\langle\alpha_{3} \mid\left[X_{1}, X_{2}\right]\right\rangle \\
& \quad=\left\langle L_{X_{1}} \alpha_{2} \mid X_{3}\right\rangle+\mathbf{d} \alpha_{1}\left(X_{3}, X_{2}\right)+\left\langle L_{X_{2}} \alpha_{3} \mid X_{1}\right\rangle-\left\langle\mathbf{d}\left\langle\alpha_{3} \mid X_{1}\right\rangle \mid X_{2}\right\rangle \\
& \quad=\left\langle L_{X_{1}} \alpha_{2} \mid X_{3}\right\rangle+\left\langle L_{X_{2}} \alpha_{3} \mid X_{1}\right\rangle+\left\langle L_{X_{3}} \alpha_{1} \mid X_{2}\right\rangle
\end{aligned}
$$

since $\mathbf{d} \alpha_{1}\left(X_{2}, X_{3}\right)=-\mathbf{d} \alpha_{1}\left(X_{3}, X_{2}\right)$, and $\mathbf{d}\left\langle\alpha_{3} \mid X_{1}\right\rangle+\mathbf{d}\left\langle\alpha_{1} \mid X_{3}\right\rangle=0$ because $\left(X_{1}, \alpha_{1}\right)$, $\left(X_{3}, \alpha_{3}\right) \in \mathcal{D}$. Thus,

$$
\begin{aligned}
& \mathcal{D} \text { is closed } \\
& \text { 出 } \\
& \left\langle L_{X_{1}} \alpha_{2} \mid X_{3}\right\rangle+\left\langle L_{X_{2}} \alpha_{3} \mid X_{1}\right\rangle+\left\langle L_{X_{3}} \alpha_{1} \mid X_{2}\right\rangle=0 \\
& \text { for all }\left(X_{1}, \alpha_{1}\right),\left(X_{2}, \alpha_{2}\right),\left(X_{3}, \alpha_{3}\right) \in \mathcal{D} \\
& \Uparrow \\
& \left\langle\mathbf{i}_{X_{1}} \mathbf{d} \alpha_{2}-\mathbf{i}_{X_{2}} \mathbf{d} \alpha_{1}+\mathbf{d}\left\langle\alpha_{2} \mid X_{1}\right\rangle \mid X_{3}\right\rangle+\left\langle\alpha_{3} \mid\left[X_{1}, X_{2}\right]\right\rangle=0 \\
& \text { for all }\left(X_{1}, \alpha_{1}\right),\left(X_{2}, \alpha_{2}\right),\left(X_{3}, \alpha_{3}\right) \in \mathcal{D} \\
& \text { 文 } \\
& \left(\left[X_{1}, X_{2}\right], \mathbf{i}_{X_{1}} \mathbf{d} \alpha_{2}-\mathbf{i}_{X_{2}} \mathbf{d} \alpha_{1}+\mathbf{d}\left\langle\alpha_{2} \mid X_{1}\right\rangle\right) \in \mathcal{D} \\
& \text { for all }\left(X_{1}, \alpha_{1}\right),\left(X_{2}, \alpha_{2}\right) \in \mathcal{D} \text {, }
\end{aligned}
$$

where the last equivalence follows from the fact that $\mathcal{D}=\mathcal{D}^{\perp}$.

REMARK 4.1. Courant [C1] uses property (4.2) as the definition of closedness (or integrability) of a generalized Dirac structure.

Closedness needs only to be checked on a set of pairs $\left(X_{i}, \alpha_{i}\right)$ which span the generalized Dirac structure $\mathcal{D}$, as follows from the following lemma.

Lemma 4.1. Consider a generalized Dirac structure $\mathcal{D}$ on a manifold $\mathcal{X}$. Let

$$
\left(X_{1}, \alpha_{1}\right), \ldots,\left(X_{n}, \alpha_{n}\right) \in \mathcal{D}
$$

and suppose that

$$
\left(\left[X_{i}, X_{j}\right], \mathbf{i}_{X_{i}} \mathbf{d} \alpha_{j}-\mathbf{i}_{X_{j}} \mathbf{d} \alpha_{i}+\mathbf{d}\left\langle\alpha_{j} \mid X_{i}\right\rangle\right) \in \mathcal{D}, \quad i, j=1, \ldots, n .
$$

Then also $\left([X, Y], \mathbf{i}_{X} \mathbf{d} \beta-\mathbf{i}_{Y} \mathbf{d} \alpha+\mathbf{d}\langle\beta \mid X\rangle\right) \in \mathcal{D}$, where

$$
(X, \alpha)=\sum_{i=1}^{n} \zeta_{i}\left(X_{i}, \alpha_{i}\right), \quad(Y, \beta)=\sum_{i=1}^{n} \eta_{i}\left(X_{i}, \alpha_{i}\right)
$$

for arbitrary $\zeta_{i}, \eta_{i} \in C^{\infty}(\mathcal{X}), i=1, \ldots, n$. 
Proof. Let $\gamma=\mathbf{i}_{X} \mathbf{d} \beta-\mathbf{i}_{Y} \mathbf{d} \alpha+\mathbf{d}\langle\beta \mid X\rangle$. A straightforward calculation then gives

$$
\begin{aligned}
& {[X, Y]=\sum_{i, j=1}^{n}\left[\zeta_{i} X_{i}\left(\eta_{j}\right) X_{j}+\zeta_{i} \eta_{j}\left[X_{i}, X_{j}\right]-\eta_{j} X_{j}\left(\zeta_{i}\right) X_{i}\right]} \\
& \gamma=\sum_{i, j=1}^{n}\left[\zeta_{i} X_{i}\left(\eta_{j}\right) \alpha_{j}+\zeta_{i} \eta_{j}\left(\mathbf{i}_{X_{i}} \mathbf{d} \alpha_{j}-\mathbf{i}_{X_{j}} \mathbf{d} \alpha_{i}+\mathbf{d}\left\langle\alpha_{j} \mid X_{i}\right\rangle\right)-\eta_{j} X_{j}\left(\zeta_{i}\right) \alpha_{i}\right] .
\end{aligned}
$$

Thus, from (4.8) it follows that $([X, Y], \gamma) \in \mathcal{D}$.

A smooth function $H \in C^{\infty}(\mathcal{X})$ is said to be admissible (see [C1]) if there exists a (smooth) vector field $X$ such that $(X, \mathbf{d} H) \in \mathcal{D}$. From the definition of the codistribution $P_{1}$ in (3.2) we see that the space of all admissible functions is given by

$$
\mathcal{A}_{\mathcal{D}}=\left\{H \in C^{\infty}(\mathcal{X}) \mid \mathbf{d} H \in P_{1}\right\} .
$$

There is a well-defined generalized Poisson bracket on $\mathcal{A}_{\mathcal{D}}$ given by the formula

$$
\left\{H_{1}, H_{2}\right\}_{\mathcal{D}}=\left\langle\mathbf{d} H_{1} \mid X_{2}\right\rangle=-\left\langle\mathbf{d} H_{2} \mid X_{1}\right\rangle,
$$

where $\left(X_{1}, \mathbf{d} H_{1}\right),\left(X_{2}, \mathbf{d} H_{2}\right) \in \mathcal{D}$. To show that $\{,\}_{\mathcal{D}}$ as defined in (4.13) is a generalized Poisson bracket is straightforward. Bilinearity of $\{,\}_{\mathcal{D}}$ follows from bilinearity of $\langle\mid\rangle$. Skew-symmetry is a consequence of (4.13). Finally, take arbitrary $\left(X_{1}, \mathbf{d} H_{1}\right),\left(X_{2}, \mathbf{d} H_{2}\right),\left(X_{3}, \mathbf{d} H_{3}\right) \in \mathcal{D}$. Then

$$
\begin{array}{r}
\left\{H_{1}, H_{2} H_{3}\right\}_{D}=-\left\langle\mathbf{d}\left(H_{2} H_{3}\right) \mid X_{1}\right\rangle=-\left\langle H_{3} \mathbf{d} H_{2}+H_{2} \mathbf{d} H_{3} \mid X_{1}\right\rangle \\
=H_{3}\left\{H_{1}, H_{2}\right\}_{\mathcal{D}}+H_{2}\left\{H_{1}, H_{3}\right\}_{\mathcal{D}}
\end{array}
$$

so $\{,\}_{\mathcal{D}}$ also satisfies the Leibniz identity. For a Dirac structure given by representation II (see Theorem 3.1), $\{,\}_{\mathcal{D}}$ is given as follows:

$$
\left\{H_{1}, H_{2}\right\}_{\mathcal{D}}(x)=\left[\frac{\partial H_{1}}{\partial x}(x)\right]^{T} J(x) \frac{\partial H_{2}}{\partial x}(x), \quad H_{1}, H_{2} \in \mathcal{A}_{\mathcal{D}} .
$$

We will now characterize closedness of a generalized Dirac structure $\mathcal{D}$ in terms of the bracket $\{,\}_{\mathcal{D}}$ and the admissible functions $\mathcal{A}_{\mathcal{D}}$. The following necessary conditions for closedness follow from Theorem 4.1.

Corollary 4.1 (cf. [C1, D2]). If $\mathcal{D}$ is closed, then

1. $G_{0}$ and $G_{1}$ are involutive distributions;

2. $\left\{H_{1}, H_{2}\right\}_{D} \in \mathcal{A}_{\mathcal{D}}$;

3. $\left\{H_{1},\left\{H_{2}, H_{3}\right\}_{\mathcal{D}}\right\}_{D}+\left\{H_{2},\left\{H_{3}, H_{1}\right\}_{\mathcal{D}}\right\}_{D}+\left\{H_{3},\left\{H_{1}, H_{2}\right\}_{\mathcal{D}}\right\}_{D}=0$ for all $H_{1}, H_{2}, H_{3} \in \mathcal{A}_{\mathcal{D}}$.

Proof.

1. Let $X_{1}, X_{2} \in G_{0}$, i.e., $\left(X_{1}, 0\right),\left(X_{2}, 0\right) \in \mathcal{D}$. Then by Theorem $4.1\left(\left[X_{1}, X_{2}\right], 0\right)$ $\in \mathcal{D}$, which means that $\left[X_{1}, X_{2}\right] \in G_{0}$. Involutivity of $G_{1}$ also follows directly from Theorem 4.1.

2. Take $H_{1}, H_{2} \in \mathcal{A}_{\mathcal{D}}$ so that $\left(X_{1}, \mathbf{d} H_{1}\right),\left(X_{2}, \mathbf{d} H_{2}\right) \in \mathcal{D}$. Then we have $\left(\left[X_{1}, X_{2}\right]\right.$, $\left.\mathbf{d}\left\langle\mathbf{d} H_{2} \mid X_{1}\right\rangle\right) \in \mathcal{D}$, which means that

$$
\mathbf{d}\left\langle\mathbf{d} H_{2} \mid X_{1}\right\rangle=\mathbf{d}\left\{H_{2}, H_{1}\right\}_{\mathcal{D}} \in P_{1} \Rightarrow\left\{H_{1}, H_{2}\right\}_{\mathcal{D}} \in \mathcal{A}_{\mathcal{D}} .
$$


3. Take $H_{1}, H_{2}, H_{3} \in \mathcal{A}_{\mathcal{D}}$ so that $\left(X_{1}, \mathbf{d} H_{1}\right),\left(X_{2}, \mathbf{d} H_{2}\right),\left(X_{3}, \mathbf{d} H_{3}\right) \in \mathcal{D}$. Then

$$
\begin{aligned}
0 & =\left\langle L_{X_{1}} \mathbf{d} H_{2} \mid X_{3}\right\rangle+\left\langle L_{X_{2}} \mathbf{d} H_{3} \mid X_{1}\right\rangle+\left\langle L_{X_{3}} \mathbf{d} H_{1} \mid X_{2}\right\rangle \\
& =\left\langle\mathbf{d}\left\langle\mathbf{d} H_{2} \mid X_{1}\right\rangle \mid X_{3}\right\rangle+\left\langle\mathbf{d}\left\langle\mathbf{d} H_{3} \mid X_{2}\right\rangle \mid X_{1}\right\rangle+\left\langle\mathbf{d}\left\langle\mathbf{d} H_{1} \mid X_{3}\right\rangle \mid X_{2}\right\rangle \\
& =\left\langle\mathbf{d}\left\{H_{2}, H_{1}\right\}_{\mathcal{D}} \mid X_{3}\right\rangle+\left\langle\mathbf{d}\left\{H_{3}, H_{2}\right\}_{\mathcal{D}} \mid X_{1}\right\rangle+\left\langle\mathbf{d}\left\{H_{1}, H_{3}\right\}_{\mathcal{D}} \mid X_{2}\right\rangle \\
& =\left\{\left\{H_{2}, H_{1}\right\}_{\mathcal{D}}, H_{3}\right\}_{\mathcal{D}}+\left\{\left\{H_{3}, H_{2}\right\}_{\mathcal{D}}, H_{1}\right\}_{\mathcal{D}}+\left\{\left\{H_{1}, H_{3}\right\}_{\mathcal{D}}, H_{2}\right\}_{\mathcal{D}} \\
& =\left\{H_{1},\left\{H_{2}, H_{3}\right\}_{\mathcal{D}}\right\}_{\mathcal{D}}+\left\{H_{2},\left\{H_{3}, H_{1}\right\}_{\mathcal{D}}\right\}_{\mathcal{D}}+\left\{H_{3},\left\{H_{1}, H_{2}\right\}_{\mathcal{D}}\right\}_{\mathcal{D}} .
\end{aligned}
$$

If in addition the codistribution $P_{1}$ (see (3.2)) is constant-dimensional, the following theorem gives necessary and sufficient conditions for closedness in terms of $\{,\}_{\mathcal{D}}$ and $\mathcal{A}_{\mathcal{D}}$.

Theorem 4.2. Consider a generalized Dirac structure $\mathcal{D}$ on a manifold $\mathcal{X}$. Let $P_{1}$ denote the codistribution on $\mathcal{X}$ defined by (3.2). Assume that $P_{1}$ is constantdimensional. Then $\mathcal{D}$ is closed if and only if the following three conditions are satisfied:

1. $G_{0}=\operatorname{ker} P_{1}$ is involutive;

2. $\left\{H_{1}, H_{2}\right\}_{\mathcal{D}} \in \mathcal{A}_{\mathcal{D}}$;

3. $\left\{H_{1},\left\{H_{2}, H_{3}\right\}_{\mathcal{D}}\right\}_{D}+\left\{H_{2},\left\{H_{3}, H_{1}\right\}_{\mathcal{D}}\right\}_{D}+\left\{H_{3},\left\{H_{1}, H_{2}\right\}_{\mathcal{D}}\right\}_{D}=0$ for all $H_{1}, H_{2}, H_{3} \in \mathcal{A}_{\mathcal{D}}$.

Proof. The necessity of these three conditions follows from Corollary 4.1 so we have to show only the sufficiency part here. First note that by using Proposition 3.1 we have that $P_{1}=\operatorname{ann} G_{0}$. Since $G_{0}=\operatorname{ker} P_{1}$ is involutive and $P_{1}$ is constantdimensional, by Frobenius's theorem in a neighborhood of any point $x_{0} \in \mathcal{X}$ there exist local coordinates $x=\left(x_{1}, \ldots, x_{n}\right)$ such that

$$
P_{1}=\operatorname{ann} G_{0}=\operatorname{span}\left\{\mathbf{d} x_{1}, \ldots, \mathbf{d} x_{n-m}\right\},
$$

where $m=\operatorname{dim} \operatorname{ker} P_{1}\left(=\operatorname{dim} G_{0}\right)$. In the following, every computation is done in such a neighborhood using local coordinates.

Take now arbitrary $\left(X_{1}, \alpha_{1}\right),\left(X_{2}, \alpha_{2}\right) \in \mathcal{D}$. Then, since $\alpha_{1}, \alpha_{2} \in P_{1}$, we have that

$$
\begin{aligned}
\alpha_{1} & =\sum_{i=1}^{n-m} \zeta_{i} \mathbf{d} x_{i}, \\
\alpha_{2} & =\sum_{i=1}^{n-m} \eta_{i} \mathbf{d} x_{i},
\end{aligned}
$$

where $\zeta_{i}, \eta_{i}$ are smooth functions. Now, let the vector fields $Y_{1}, \ldots, Y_{n-m}$ be such that

$$
\left(Y_{i}, \mathbf{d} x_{i}\right) \in \mathcal{D}, \quad 1 \leq i \leq n-m .
$$

Since also $\left(X_{1}, \alpha_{1}\right) \in \mathcal{D}$ it follows that

$$
\left\langle\mathbf{d} x_{k} \mid X_{1}\right\rangle+\left\langle\sum_{i=1}^{n-m} \zeta_{i} \mathbf{d} x_{i} \mid Y_{k}\right\rangle=0
$$

so

$$
\left\langle\mathbf{d} x_{k} \mid X_{1}\right\rangle=-\sum_{i=1}^{n-m} \zeta_{i}\left\{x_{i}, x_{k}\right\}_{\mathcal{D}}
$$


for $1 \leq k \leq n-m$. Define the vector fields $Z_{1}, Z_{2}$ as

$$
\begin{aligned}
& Z_{1}=X_{1}-\sum_{i=1}^{n-m} \zeta_{i} Y_{i}, \\
& Z_{2}=X_{2}-\sum_{i=1}^{n-m} \eta_{i} Y_{i} .
\end{aligned}
$$

Then

$$
\begin{aligned}
\left\langle\mathbf{d} x_{k} \mid Z_{1}\right\rangle & =\left\langle\mathbf{d} x_{k} \mid X_{1}-\sum_{i=1}^{n-m} \zeta_{i} Y_{i}\right\rangle \\
& =-\sum_{i=1}^{n-m} \zeta_{i}\left\{x_{i}, x_{k}\right\}_{\mathcal{D}}-\sum_{i=1}^{n-m} \zeta_{i}\left\{x_{k}, x_{i}\right\}_{\mathcal{D}} \\
& =0
\end{aligned}
$$

for all $1 \leq k \leq n-m$ since $\left\{x_{i}, x_{k}\right\}_{\mathcal{D}}=-\left\{x_{k}, x_{i}\right\}_{\mathcal{D}}$. This means that $Z_{1} \in \operatorname{ker} P_{1}=G_{0}$ and

$$
\begin{aligned}
X_{1} & =\sum_{i=1}^{n-m} \zeta_{i} Y_{i}+Z_{1}, \\
X_{2} & =\sum_{i=1}^{n-m} \eta_{i} Y_{i}+Z_{2},
\end{aligned}
$$

where $Z_{1}, Z_{2} \in G_{0}$.

Now we want to calculate the term

$$
\alpha_{12}=\mathbf{i}_{X_{1}} \mathbf{d} \alpha_{2}-\mathbf{i}_{X_{2}} \mathbf{d} \alpha_{1}+\mathbf{d}\left\langle\alpha_{2} \mid X_{1}\right\rangle .
$$

We have $\mathbf{d} \alpha_{2}=\mathbf{d}\left(\sum_{i=1}^{n-m} \eta_{i} \mathbf{d} x_{i}\right)=\sum_{i=1}^{n-m} \mathbf{d} \eta_{i} \wedge \mathbf{d} x_{i}$, so

$$
\begin{aligned}
\mathbf{i}_{X_{1}} \mathbf{d} \alpha_{2} & =\mathbf{i}_{X_{1}}\left(\sum_{i=1}^{n-m} \mathbf{d} \eta_{i} \wedge \mathbf{d} x_{i}\right) \\
& =\sum_{i=1}^{n-m}\left[\mathbf{i}_{X_{1}} \mathbf{d} \eta_{i} \wedge \mathbf{d} x_{i}-\mathbf{d} \eta_{i} \wedge \mathbf{i}_{X_{1}} \mathbf{d} x_{i}\right] \\
& =\sum_{i=1}^{n-m}\left[\left\langle\mathbf{d} \eta_{i} \mid \sum_{j=1}^{n-m} \zeta_{j} Y_{j}+Z_{1}\right\rangle \mathbf{d} x_{i}-\left\langle\mathbf{d} x_{i} \mid \sum_{j=1}^{n-m} \zeta_{j} Y_{j}+Z_{1}\right\rangle \mathbf{d} \eta_{i}\right] \\
& =\sum_{i, j=1}^{n-m}\left[\zeta_{j} Y_{j}\left(\eta_{i}\right) \mathbf{d} x_{i}-\zeta_{j}\left\{x_{i}, x_{j}\right\}_{D} \mathbf{d} \eta_{i}\right]+\sum_{i=1}^{n-m} Z_{1}\left(\eta_{i}\right) \mathbf{d} x_{i},
\end{aligned}
$$

where we used the fact that $\left\langle\mathbf{d} x_{i} \mid Z_{1}\right\rangle=0$ since $\mathbf{d} x_{i} \in \operatorname{ann} G_{0}$. Similarly we obtain

$$
\mathbf{i}_{X_{2}} \mathbf{d} \alpha_{1}=\sum_{i, j=1}^{n-m}\left[\eta_{j} Y_{j}\left(\zeta_{i}\right) \mathbf{d} x_{i}-\eta_{j}\left\{x_{i}, x_{j}\right\}_{\mathcal{D}} \mathbf{d} \zeta_{i}\right]+\sum_{i=1}^{n-m} Z_{2}\left(\zeta_{i}\right) \mathbf{d} x_{i} .
$$


Moreover,

$$
\begin{aligned}
\mathbf{d}\left\langle\alpha_{2} \mid X_{1}\right\rangle & =\mathbf{d}\left\langle\sum_{i=1}^{n-m} \eta_{i} \mathbf{d} x_{i} \mid \sum_{j=1}^{n-m} \zeta_{j} Y_{j}+Z_{1}\right\rangle \\
& =\mathbf{d}\left[\sum_{i, j=1}^{n-m} \eta_{i} \zeta_{j}\left\langle\mathbf{d} x_{i} \mid Y_{j}\right\rangle\right] \\
& =\mathbf{d}\left[\sum_{i, j=1}^{n-m} \eta_{i} \zeta_{j}\left\{x_{i}, x_{j}\right\}_{\mathcal{D}}\right] \\
& =\sum_{i, j=1}^{n-m}\left[\eta_{i} \zeta_{j} \mathbf{d}\left\{x_{i}, x_{j}\right\}_{\mathcal{D}}+\left\{x_{i}, x_{j}\right\}_{\mathcal{D}}\left(\zeta_{j} \mathbf{d} \eta_{i}+\eta_{i} \mathbf{d} \zeta_{j}\right)\right] \\
& =\sum_{i, j=1}^{n-m}\left[\eta_{i} \zeta_{j} \mathbf{d}\left\{x_{i}, x_{j}\right\}_{\mathcal{D}}+\left\{x_{i}, x_{j}\right\}_{\mathcal{D}}\left(\zeta_{j} \mathbf{d} \eta_{i}-\eta_{j} \mathbf{d} \zeta_{i}\right)\right]
\end{aligned}
$$

where the last equation follows from skew-symmetry of $\{,\}_{\mathcal{D}}$. Inserting (4.30), (4.31), and (4.32) in (4.29) gives

$$
\begin{aligned}
\alpha_{12}= & \mathbf{i}_{X_{1}} \mathbf{d} \alpha_{2}-\mathbf{i}_{X_{2}} \mathbf{d} \alpha_{1}+\mathbf{d}\left\langle\alpha_{2} \mid X_{1}\right\rangle \\
= & \sum_{i, j=1}^{n-m}\left[\left(\zeta_{j} Y_{j}\left(\eta_{i}\right)-\eta_{j} Y_{j}\left(\zeta_{i}\right)\right) \mathbf{d} x_{i}+\eta_{i} \zeta_{j} \mathbf{d}\left\{x_{i}, x_{j}\right\}_{\mathcal{D}}\right] \\
& +\sum_{i=1}^{n-m}\left(Z_{1}\left(\eta_{i}\right)-Z_{2}\left(\zeta_{i}\right)\right) \mathbf{d} x_{i} .
\end{aligned}
$$

From (4.33) we immediately see that $\alpha_{12} \in P_{1}$ since $\mathbf{d}\left\{x_{i}, x_{j}\right\}_{\mathcal{D}} \in P_{1}$ when $1 \leq i, j \leq$ $n-m$.

Now we have to take a closer look at the term $\left[X_{1}, X_{2}\right]$. A direct calculation yields

$$
\begin{aligned}
& {\left[X_{1}, X_{2}\right]=\left[\sum_{i=1}^{n-m} \zeta_{i} Y_{i}+Z_{1}, \sum_{j=1}^{n-m} \eta_{j} Y_{j}+Z_{2}\right] } \\
= & \sum_{i, j=1}^{n-m}\left\{\left(\zeta_{j} Y_{j}\left(\eta_{i}\right)-\eta_{j} Y_{j}\left(\zeta_{i}\right)\right) Y_{i}+\eta_{i} \zeta_{j}\left[Y_{j}, Y_{i}\right]\right\}+\sum_{i=1}^{n-m}\left(Z_{1}\left(\eta_{i}\right)-Z_{2}\left(\zeta_{i}\right)\right) Y_{i}+Z_{12},
\end{aligned}
$$

where the vector field $Z_{12}$ is given by

$$
Z_{12}=\sum_{i=1}^{n-m}\left(\zeta_{i}\left[Y_{i}, Z_{2}\right]-\eta_{i}\left[Y_{i}, Z_{1}\right]\right)+\left[Z_{1}, Z_{2}\right]
$$

Now take arbitrary $Z \in G_{0}$ and consider $\mathbf{d}\left\{x_{i}, x_{j}\right\}_{\mathcal{D}} \in \operatorname{ann} G_{0}$ for $1 \leq i, j \leq n-m$. 
Then

$$
\begin{aligned}
0 & =\left\langle\mathbf{d}\left\{x_{i}, x_{j}\right\}_{\mathcal{D}} \mid Z\right\rangle \\
& =-Z\left(\left\{x_{j}, x_{i}\right\}_{D}\right) \\
& =-Z\left(Y_{i}\left(x_{j}\right)\right) \\
& =-Y_{i}\left(Z\left(x_{j}\right)\right)+\left[Y_{i}, Z\right]\left(x_{j}\right) \\
& =\left\langle\mathbf{d} x_{j} \mid\left[Y_{i}, Z\right]\right\rangle
\end{aligned}
$$

for all $1 \leq i, j \leq n-m$, which means that $\left[Y_{i}, Z\right] \in \operatorname{ker} P_{1}=G_{0}$ for all $1 \leq i \leq n-m$. Since $G_{0}$ is involutive we immediately see from (4.35) that also $Z_{12} \in G_{0}$.

Now we want to show that $\left(\left[Y_{j}, Y_{i}\right], \mathbf{d}\left\{x_{i}, x_{j}\right\}_{\mathcal{D}}\right) \in \mathcal{D}$. We know that $\left\{x_{i}, x_{j}\right\}_{\mathcal{D}} \in$ $\mathcal{A}_{\mathcal{D}}$, which means that there exist vector fields $Y_{i j}$ such that $\left(Y_{i j}, \mathbf{d}\left\{x_{i}, x_{j}\right\}_{\mathcal{D}}\right) \in \mathcal{D}$, $i, j=1, \ldots, n-m$. Then

$$
\begin{aligned}
\left\langle\mathbf{d} x_{k} \mid\left[Y_{j}, Y_{i}\right]-Y_{i j}\right\rangle & =\left[Y_{j}, Y_{i}\right]\left(x_{k}\right)-Y_{i j}\left(x_{k}\right) \\
& =Y_{j}\left(\left\langle\mathbf{d} x_{k} \mid Y_{i}\right\rangle\right)-Y_{i}\left(\left\langle\mathbf{d} x_{k} \mid Y_{j}\right\rangle\right)-\left\langle\mathbf{d} x_{k} \mid Y_{i j}\right\rangle \\
& =\left\langle\mathbf{d}\left\{x_{k}, x_{i}\right\}_{\mathcal{D}} \mid Y_{j}\right\rangle-\left\langle\mathbf{d}\left\{x_{k}, x_{j}\right\}_{\mathcal{D}} \mid Y_{i}\right\rangle-\left\{x_{k},\left\{x_{i}, x_{j}\right\}_{\mathcal{D}}\right\}_{\mathcal{D}} \\
& =\left\{\left\{x_{k}, x_{i}\right\}_{\mathcal{D}}, x_{j}\right\}_{\mathcal{D}}-\left\{\left\{x_{k}, x_{j}\right\}_{\mathcal{D}}, x_{i}\right\}_{\mathcal{D}}-\left\{x_{k},\left\{x_{i}, x_{j}\right\}_{\mathcal{D}}\right\}_{\mathcal{D}} \\
& =\left\{x_{j},\left\{x_{i}, x_{k}\right\}_{\mathcal{D}}\right\}_{\mathcal{D}}+\left\{x_{i},\left\{x_{k}, x_{j}\right\}_{\mathcal{D}}\right\}_{\mathcal{D}}+\left\{x_{k},\left\{x_{j}, x_{i}\right\}_{\mathcal{D}}\right\}_{\mathcal{D}} \\
& =0
\end{aligned}
$$

when $1 \leq i, j, k \leq n-m$, which means that $\left[Y_{j}, Y_{i}\right]-Y_{i j} \in \operatorname{ker} P_{1}=G_{0}$. Thus,

$$
\left(\left[Y_{j}, Y_{i}\right], \mathbf{d}\left\{x_{i}, x_{j}\right\}\right) \in \mathcal{D}, \quad i, j=1, \ldots, n-m,
$$

and by inspection of (4.33) and (4.34) we see that $\left(\left[X_{1}, X_{2}\right], \alpha_{12}\right) \in \mathcal{D}$, and closedness of $\mathcal{D}$ follows from Theorem 4.1 .

In the following we will explicitly characterize closedness in the three different representations of a Dirac structure.

TheOrem 4.3 (representation I). Consider a generalized Dirac structure $\mathcal{D}$ on a manifold $\mathcal{X}$ given locally in representation I (see (3.8), (3.9)). Define $\left(X_{i}, \alpha_{i}\right) \in \mathcal{D}$ in local coordinates by

$$
\begin{aligned}
X_{i} & =E_{i}^{T}(x), \\
\alpha_{i} & =-F_{i}^{T}(x),
\end{aligned}
$$

where $E_{i}^{T}(x)$ and $F_{i}^{T}(x)$ denote the ith column of the matrices $E^{T}(x)$ and $F^{T}(x)$, respectively. Then $\mathcal{D}$ is closed if and only if

$$
\begin{aligned}
\left(\left[X_{i}, X_{j}\right], \mathbf{i}_{X_{i}} \mathbf{d} \alpha_{j}-\mathbf{i}_{X_{j}} \mathbf{d} \alpha_{i}+\mathbf{d}\left\langle\alpha_{j} \mid X_{i}\right\rangle\right) & \in \mathcal{D}(x) \\
\text { for all } x & \in \mathcal{X}, i, j=1, \ldots, n .
\end{aligned}
$$

Proof. The proof follows from (3.27), Theorem 4.1, and Lemma 4.1.

TheOREM 4.4 (representation II). Let $\mathcal{X}$ be an n-dimensional manifold. Let $G$ be a constant-dimensional distribution on $\mathcal{X}$, and $J(x): T_{x}^{*} \mathcal{X} \rightarrow T_{x} \mathcal{X}, x \in \mathcal{X}$, be a skew-symmetric vector bundle map. Moreover, let $\{$,$\} denote the generalized$ Poisson bracket corresponding to J. Then the generalized Dirac structure given by (see Theorem 3.1)

$$
\mathcal{D}=\left\{(X, \alpha) \in T \mathcal{X} \oplus T^{*} \mathcal{X} \mid X(x)-J(x) \alpha(x) \in G(x), x \in \mathcal{X}, \alpha \in \text { ann } G\right\}
$$

is closed if and only if 
1. $G$ is involutive;

2. $\left\{H_{1}, H_{2}\right\} \in \mathcal{A}_{\mathcal{D}}$;

3. $\left\{H_{1},\left\{H_{2}, H_{3}\right\}\right\}+\left\{H_{2},\left\{H_{3}, H_{1}\right\}\right\}+\left\{H_{3},\left\{H_{1}, H_{2}\right\}\right\}=0$ for all $H_{1}, H_{2}, H_{3} \in \mathcal{A}_{\mathcal{D}}=\left\{H \in C^{\infty}(\mathcal{X}) \mid \mathbf{d} H \in\right.$ ann $\left.G\right\}$.

Proof. The result follows from Theorem 4.2 using the facts that $G_{0}=G$ and that $\left\{H_{1}, H_{2}\right\}_{\mathcal{D}}=\left\{H_{1}, H_{2}\right\}$ for all $H_{1}, H_{2} \in \mathcal{A}_{\mathcal{D}}$.

THEOREM 4.5 (representation III). Let $\mathcal{X}$ be an $n$-dimensional manifold. Let $P$ be a constant-dimensional codistribution on $\mathcal{X}$, and $\omega(x): T_{x} \mathcal{X} \rightarrow T_{x}^{*} \mathcal{X}, x \in \mathcal{X}$, be a skew-symmetric vector bundle map. Then the generalized Dirac structure given by (see Theorem 3.2)

$$
\mathcal{D}=\left\{(X, \alpha) \in T \mathcal{X} \oplus T^{*} \mathcal{X} \mid \alpha(x)-\omega(x) X(x) \in P(x), x \in \mathcal{X}, X \in \text { ker } P\right\}
$$

is closed if and only if

1. $\operatorname{ker} P$ is involutive;

2. $\mathbf{d} \omega\left(X_{1}, X_{2}, X_{3}\right)=0$ for all $X_{1}, X_{2}, X_{3} \in \operatorname{ker} P$.

Proof. Let $\left(X_{1}, \alpha_{1}\right),\left(X_{2}, \alpha_{2}\right) \in \mathcal{D}$, i.e.,

$$
\alpha_{i}=\mathbf{i}_{X_{i}} \omega+p_{i}, \quad p_{i} \in P, X_{i} \in \operatorname{ker} P, \quad i=1,2 .
$$

Define as in (4.29) the one-form $\alpha_{12}=\mathbf{i}_{X_{1}} \mathbf{d} \alpha_{2}-\mathbf{i}_{X_{2}} \mathbf{d} \alpha_{1}+\mathbf{d}\left\langle\alpha_{2} \mid X_{1}\right\rangle$. Now, using Cartan's magic formula, we get

$$
\begin{aligned}
\operatorname{di}_{X_{1}} \omega & =L_{X_{1}} \omega-\mathbf{i}_{X_{1}} \mathbf{d} \omega \\
\operatorname{di}_{X_{1}} \mathbf{i}_{X_{2}} \omega & =L_{X_{1}} \mathbf{i}_{X_{2}} \omega-\mathbf{i}_{X_{1}} L_{X_{2}} \omega+\mathbf{i}_{X_{1}} \mathbf{i}_{X_{2}} \mathbf{d} \omega
\end{aligned}
$$

for all vector fields $X_{1}, X_{2}$ on $\mathcal{X}$. Hence

$$
\begin{aligned}
\alpha_{12} & =\mathbf{i}_{X_{1}} \mathbf{d} \alpha_{2}-\mathbf{i}_{X_{2}} \mathbf{d} \alpha_{1}+\mathbf{d}\left\langle\alpha_{2} \mid X_{1}\right\rangle \\
& =\mathbf{i}_{X_{1}} \mathbf{d}\left(\mathbf{i}_{X_{2}} \omega+p_{2}\right)-\mathbf{i}_{X_{2}} \mathbf{d}\left(\mathbf{i}_{X_{1}} \omega+p_{1}\right)+\mathbf{d} \mathbf{i}_{X_{1}}\left(\mathbf{i}_{X_{2}} \omega+p_{2}\right) \\
& =\mathbf{i}_{X_{1}} \mathbf{d} \mathbf{i}_{X_{2}} \omega+\mathbf{i}_{X_{1}} \mathbf{d} p_{2}-\mathbf{i}_{X_{2}} \mathbf{d} \mathbf{i}_{X_{1}} \omega-\mathbf{i}_{X_{2}} \mathbf{d} p_{1}+\mathbf{d i}_{X_{1}} \mathbf{i}_{X_{2}} \omega+\mathbf{d} \mathbf{i}_{X_{1}} p_{2} \\
& =-\mathbf{i}_{X_{2}} L_{X_{1}} \omega+L_{X_{1}} \mathbf{i}_{X_{2}} \omega+\mathbf{i}_{X_{1}} \mathbf{d} p_{2}-\mathbf{i}_{X_{2}} \mathbf{d} p_{1}+\mathbf{i}_{X_{2}} \mathbf{i}_{X_{1}} \mathbf{d} \omega \\
& =\mathbf{i}_{\left.X_{1}, X_{2}\right]} \omega+\mathbf{i}_{X_{1}} \mathbf{d} p_{2}-\mathbf{i}_{X_{2}} \mathbf{d} p_{1}+\mathbf{i}_{X_{2}} \mathbf{i}_{X_{1}} \mathbf{d} \omega
\end{aligned}
$$

since $\mathbf{i}_{\left[X_{1}, X_{2}\right]} \omega=L_{X_{1}} \mathbf{i}_{X_{2}} \omega-\mathbf{i}_{X_{2}} L_{X_{1}} \omega$. Thus, using Theorem 4.1 and the definition of $\mathcal{D}$, we have that

$$
\begin{aligned}
& \mathcal{D} \text { is closed } \\
& \mathbb{1} \\
& \left(\left[X_{1}, X_{2}\right], \mathbf{i}_{\left[X_{1}, X_{2}\right]} \omega+\mathbf{i}_{X_{1}} \mathbf{d} p_{2}-\mathbf{i}_{X_{2}} \mathbf{d} p_{1}+\mathbf{i}_{X_{2}} \mathbf{i}_{X_{1}} \mathbf{d} \omega\right) \in \mathcal{D} \\
& \text { for all } p_{1}, p_{2} \in P \text {, for all } X_{1}, X_{2} \in \operatorname{ker} P \text {. } \\
& \mathbb{1} \\
& \left.\begin{array}{l}
{\left[X_{1}, X_{2}\right] \in \operatorname{ker} P} \\
\mathbf{i}_{X_{1}} \mathbf{d} p_{2}-\mathbf{i}_{X_{2}} \mathbf{d} p_{1}+\mathbf{i}_{X_{2}} \mathbf{i}_{X_{1}} \mathbf{d} \omega \in P
\end{array}\right\} \text { for all } p_{1}, p_{2} \in P \text {, for all } X_{1}, X_{2} \in \operatorname{ker} P .
\end{aligned}
$$

Now, if $P$ is a constant-dimensional codistribution and $\operatorname{ker} P$ is involutive, it follows that for every $p \in P$ there exists $\bar{p} \in P$ and a one-form $\eta$ such that $\mathbf{d} p=\eta \wedge \bar{p}$. Thus, $\mathbf{i}_{X} \mathbf{d} p=\eta(X) \bar{p} \in P$ for all $X \in \operatorname{ker} P$. Moreover, $\mathbf{i}_{X_{2}} \mathbf{i}_{X_{1}} \mathbf{d} \omega\left(X_{3}\right)=\mathbf{d} \omega\left(X_{1}, X_{2}, X_{3}\right)$ which means that $\mathbf{i}_{X_{2}} \mathbf{i}_{X_{1}} \mathbf{d} \omega \in P$ if and only if $\mathbf{d} \omega\left(X_{1}, X_{2}, X_{3}\right)=0$ for all $X_{3} \in \operatorname{ker} P$ since $P$ is constant-dimensional. 
REMARK 4.2. In $[\mathrm{C} 1]$ it is shown that closedness of $D$ implies condition 2 in Theorem 4.5.

We will now apply the above theory to mechanical systems with kinematic constraints (see Example 3.2).

Proposition 4.1. Consider the mechanical system with kinematic constraints $A^{T}(q) \dot{q}=0$ as given in Example 3.2. Let $\{$,$\} denote the Poisson bracket defined$ (locally) by the structure matrix $J$. Then the following statements are equivalent:

1. $\mathcal{D}_{A}$ is closed;

2. the constraints (3.21) are holonomic;

3. $\mathbf{d}\left\{H_{1}, H_{2}\right\} \in$ ann $G$ for all $H_{1}, H_{2}$ such that $\mathbf{d} H_{1}, \mathbf{d} H_{2} \in$ ann $G$.

Proof. $1 \Leftrightarrow 2$ : From Theorem 4.5 it follows that $\mathcal{D}_{A}$ is closed if and only if ker $P$ is involutive which is equivalent to the constraints $(3.21)$ being holonomic. $1 \Leftrightarrow 3$ : This follows from Theorem 4.4 since $G$ is involutive and $\{$,$\} satisfies the Jacobi identity$ in this case.

The next proposition gives an interesting interpretation of closedness of generalized Dirac structures that come up in connection with Lie-Poisson structures.

Proposition 4.2. Let $G$ be any n-dimensional Lie group (e.g., SE(3)), with Lie algebra $g$, and the dual Lie algebra $g^{*}$ with the Lie-Poisson bracket $\{$,$\} . Consider$ a constant distribution on $g^{*}$, that is a linear subspace $\mathcal{V} \subset g^{*}$. Define the Dirac structure $\mathcal{D}$ on $g^{*}$ as

$$
\mathcal{D}=\left\{(X, \alpha) \in T g^{*} \oplus T^{*} g^{*} \mid X(x)-J(x) \alpha(x) \in \mathcal{V}, \alpha(x) \in \mathcal{V}^{\perp}, x \in g^{*}\right\},
$$

where $J(x)$ is the structure matrix of the Lie-Poisson bracket $\{$,$\} . Then \mathcal{D}$ is closed if and only if $\mathcal{V}^{\perp} \subset g$ is a subalgebra.

Proof. The proof follows more or less directly from results obtained in [MR, p. 287].

EXAMPLE $4.1\left(\mathcal{X}=s e^{*}(3) \simeq \mathbb{R}^{6}\right)$. The motion of a rigid body with respect to a body-fixed rotation reference frame in the center of mass is given (in the absence of gravity) by

$$
\begin{aligned}
M \dot{\omega}+\omega \times M \omega & =\tau, \\
m \dot{v}+\omega \times m v & =F,
\end{aligned}
$$

where $v, \omega \in \mathbb{R}^{3}$ are, respectively, the linear and the angular velocities, $M$ is the inertia tensor, and $\tau, F \in \mathbb{R}^{3}$ are, respectively, the torques and the forces. By defining $\Pi, p \in \mathbb{R}^{3}$ as

$$
\begin{aligned}
\Pi & =M \omega, \quad \Pi=\left[\Pi_{x}, \Pi_{y}, \Pi_{z}\right]^{T}, \\
p & =m v, \quad p=\left[p_{x}, p_{y}, p_{z}\right]^{T}
\end{aligned}
$$

and the Hamiltonian $H(\Pi, p)$ as

$$
H(\Pi, p)=\frac{1}{2} \Pi^{T} M^{-1} \Pi+\frac{1}{2 m} p^{T} p,
$$

it follows that (4.49) and (4.50) can be written as

$$
\left[\begin{array}{c}
\dot{\Pi} \\
\dot{p}
\end{array}\right]=\underbrace{\left[\begin{array}{cc}
S(\Pi) & S(p) \\
S(p) & 0
\end{array}\right]}_{J(\Pi, p)}\left[\begin{array}{c}
\frac{\partial H}{\partial \Pi} \\
\frac{\partial H}{\partial p}
\end{array}\right]+\left[\begin{array}{c}
\tau \\
F
\end{array}\right] .
$$


Here $\Pi=\left[\Pi_{x}, \Pi_{y}, \Pi_{z}\right]^{T}$ and $p=\left[p_{x}, p_{y}, p_{z}\right]^{T}$ are the body angular and linear momentum, respectively. $S(\cdot)$ is defined by $S(a) b=a \times b$ for $a, b \in \mathbb{R}^{3} . J(\Pi, p)$ is the structure matrix of the Lie-Poisson bracket on $\mathcal{X}=s e^{*}(3) \simeq \mathbb{R}^{6}$.

Assume that the following constraints are imposed on the system:

$$
p_{y}=p_{z}=0 .
$$

Let $e_{x}=\left[\begin{array}{lll}1 & 0 & 0\end{array}\right]^{T}, e_{y}=\left[\begin{array}{lll}0 & 1 & 0\end{array}\right]^{T}, e_{z}=\left[\begin{array}{lll}0 & 0 & 1\end{array}\right]^{T}$. Then

$$
\mathcal{V}^{\perp}=\operatorname{ker}\left[\begin{array}{cc}
0 & 0 \\
e_{y} & e_{z}
\end{array}\right]
$$

which is not a subalgebra of se $(3) \simeq \mathbb{R}^{6}$ (see, e.g., $\left.[\mathrm{MR}]\right)$. Hence, the corresponding generalized Dirac structure is not closed in this case. However, if the additional constraint $p_{x}=0$ is imposed on the system (fixed center of mass), it is easy to see that closedness of the corresponding generalized Dirac structure follows.

Similarly to the case when the Jacobi-identity is satisfied for a generalized Poisson structure, one can show that if the closedness condition (4.1) is satisfied for a generalized Dirac structure then there exist local canonical coordinates around any regular point in which the geometric picture simplifies considerably (see Proposition 4.1.2 in [C1]). In our context (i.e., for generalized Dirac structures arising from physical systems) constant-dimensionality of the codistribution $P_{1}$ is often a reasonable assumption. Thus, in the next proposition we will draw attention to the existence and construction of canonical coordinates for Dirac structures that may be given in representation II (cf. Theorem 3.1). In essence, the proof of this proposition comes down to using Frobenius's theorem and a generalized version of Darboux's theorem and proceeds along the same general line as the proof of Proposition 4.1.2 in [C1]. However, we show directly how local canonical coordinates may be found for a Dirac structure in representation II. In addition, we show more explicitly where the three necessary conditions in Corollary 4.1 come into play which is interesting in itself.

Proposition 4.3. Let $\mathcal{D}$ be a generalized Dirac structure on an $n$-dimensional manifold $\mathcal{X}$. Assume that the codistribution $P_{1}$ (see (3.2)) is constant-dimensional so that $\mathcal{D}$ can always be given in representation II as follows:

$$
\mathcal{D}=\left\{(X, \alpha) \in T \mathcal{X} \oplus T^{*} \mathcal{X} \mid X(x)-J(x) \alpha(x) \in G_{0}(x), x \in \mathcal{X}, \alpha \in \text { ann } G_{0}\right\},
$$

where $J(x): T_{x}^{*} \mathcal{X} \rightarrow T_{x} \mathcal{X}, x \in \mathcal{X}$, is a skew-symmetric vector bundle map. Then, if $\mathcal{D}$ is closed, there exist around every regular point $x_{0} \in \mathcal{X}$ local canonical coordinates

$$
(q, p, r, s)=\left(q_{1}, \ldots, q_{k}, p_{1}, \ldots, p_{k}, r_{1}, \ldots, r_{l}, s_{1}, \ldots, s_{m}\right), \quad 2 k+l+m=n
$$

for $\mathcal{X}$ in which $J(x)$ and $G_{0}$ take the simple form

$$
J(x)=\left[\begin{array}{cccc}
0 & I_{k} & 0 & * \\
-I_{k} & 0 & 0 & * \\
0 & 0 & 0 & * \\
* & * & * & *
\end{array}\right], \quad G_{0}=\operatorname{span}\left\{\frac{\partial}{\partial s_{1}}, \ldots, \frac{\partial}{\partial s_{m}}\right\},
$$

where $*$ denotes unspecified elements, $m=n-\operatorname{dim} P_{1}$ and $l=n-\operatorname{dim} G_{1}\left(x_{0}\right)$.

Conversely, if $\mathcal{D}$ is given by (4.57), (4.58) in a neighborhood of $x_{0} \in \mathcal{X}$, then $\mathcal{D}$ is closed in this neighborhood. 
Proof. If $\mathcal{D}$ is closed, it follows from condition 1 in Corollary 4.1 that $G_{0}$ is involutive. Since $P_{1}=\operatorname{ann} G_{0}$ is constant-dimensional, also $G_{0}$ is constant-dimensional with dimension equal to $m$. Thus, by Frobenius' theorem in a neighborhood $N_{x_{0}}$ of any point $x_{0} \in \mathcal{X}$ there exist local coordinates $(y, s)=\left(y_{1}, \ldots, y_{n-m}, s_{1}, \ldots, s_{m}\right)$, such that

$$
G_{0}=\operatorname{span}\left\{\frac{\partial}{\partial s_{1}}, \ldots, \frac{\partial}{\partial s_{m}}\right\}
$$

and

$$
P_{1}=\operatorname{ann} G_{0}=\operatorname{span}\left\{\mathbf{d} y_{1}, \ldots, \mathbf{d} y_{n-m}\right\} .
$$

Now, $\{,\}_{\mathcal{D}}$ is given in terms of $J(x)$ as follows:

$$
\{F, G\}_{\mathcal{D}}(x)=\left[\frac{\partial F}{\partial x}(x)\right]^{T} J(x) \frac{\partial G}{\partial x}(x)
$$

for all $F, G \in C^{\infty}(\mathcal{X})$ such that $\mathbf{d} F, \mathbf{d} G \in \operatorname{ann} G_{0}$. Moreover, since $\mathcal{D}$ is closed, it follows from condition 2 in Corollary 4.1 that

$$
\mathbf{d}\left\{y_{i}, y_{j}\right\}_{\mathcal{D}} \in \operatorname{ann} G_{0}, \quad i, j=1, \ldots, n-m,
$$

which means that

$$
\frac{\partial\left\{y_{i}, y_{j}\right\}_{D}}{\partial s_{k}}=0, \quad k=1, \ldots, m, i, j=1, \ldots, n-m .
$$

Hence, $J(x)$ takes the following form in the local coordinates $(y, s)$ :

$$
J(y, s)=\left[\begin{array}{cc}
\bar{J}(y) & * \\
* & *
\end{array}\right]
$$

where $\bar{J}(y)=\left[\left\{y_{i}, y_{j}\right\}_{\mathcal{D}}\right]$ is the $(n-m) \times(n-m)$ upper-left submatrix of $J(y, s)$. In addition, the distribution $G_{1}$ is given locally in the coordinates $(y, s)$ as

$$
G_{1}(y, s)=\operatorname{Im}\left[\begin{array}{cc}
\bar{J}(y) & 0 \\
0 & I_{m}
\end{array}\right] .
$$

If $x_{0} \in \mathcal{X}$ is a regular point, then $G_{1}$ is by definition constant-dimensional in a neighborhood of $x_{0}$ which implies that $\bar{J}(y)$ has constant rank $2 k=n-(l+m)$ in a neighborhood $\hat{N}_{x_{0}} \subset N_{x_{0}}$ of $x_{0}$. Define (without loss of generality) the submanifold $\mathcal{Y} \subset \mathcal{X}$ as

$$
\mathcal{Y}=\left\{(y, s) \in \hat{N}_{x_{0}} \mid s=s\left(x_{0}\right)\right\} .
$$

$y=\left(y_{1}, \ldots, y_{n-m}\right)$ are local coordinates for $\mathcal{Y}$ around $y_{0}=y\left(x_{0}\right)$. Since $\mathcal{D}$ is closed, it follows from condition 3 in Corollary 4.1 that $\{,\}_{\mathcal{D}}$ defines a Poisson structure on $\mathcal{Y}$ with structure matrix $\bar{J}(y)$. Now, using the fact that $\bar{J}(y)$ has constant rank $2 k \leq n-m$ for all $y \in \mathcal{Y}$, it follows from Theorem 6.22 in [O] (called the generalized Darboux's theorem; see also [W]), that around $y_{0} \in \mathcal{Y}$ there exist local coordinates $(q, p, r)=\left(q_{1}, \ldots, q_{k}, p_{1}, \ldots, p_{k}, r_{1}, \ldots, r_{l}\right)$ in which $\bar{J}(y)$ takes the form

$$
\bar{J}(p, q, r)=\left[\begin{array}{ccc}
0 & I_{k} & 0 \\
-I_{k} & 0 & 0 \\
0 & 0 & 0
\end{array}\right] .
$$


Now $(q, p, r, s)$ are local coordinates for $\mathcal{X}$ around $x_{0} \in \mathcal{X}$ in which $J(x)$ and $G_{0}$ take the simple form (4.58).

Conversely, it is easy to check that a generalized Dirac structure given by (4.57), (4.58) in a neighborhood of $x_{0} \in \mathcal{X}$, satisfies the sufficient conditions for closedness as given in Theorem 4.2 in this neighborhood.

The equations of an implicit generalized Hamiltonian system corresponding to the local representation (4.57), (4.58) and a Hamiltonian $H$ take the form

$$
\begin{aligned}
\dot{q} & =\frac{\partial H}{\partial p}(q, p, r, s), \\
\dot{p} & =-\frac{\partial H}{\partial q}(q, p, r, s), \\
\dot{r} & =0, \\
0 & =\frac{\partial H}{\partial s}(q, p, r, s) .
\end{aligned}
$$

Comparing (4.68) with (2.7) we see that while (2.7) makes explicit the conserved quantities, (4.68) also makes explicit the algebraic constraints

$$
\begin{aligned}
0 & =\frac{\partial H}{\partial s_{1}}(q, p, r, s), \\
& \vdots \\
0 & =\frac{\partial H}{\partial s_{m}}(q, p, r, s) .
\end{aligned}
$$

If $H$ is nondegenerate in the energy-variables $s_{1}, \ldots, s_{m}$, that is,

$$
\operatorname{rank}\left[\frac{\partial^{2} H}{\partial s_{i} \partial s_{j}}\right]=m
$$

then by the implicit function theorem one may locally express the variables $s_{1}, \ldots, s_{m}$ as functions of $q, p, r$, i.e., $s_{i}=s_{i}(q, p, r), i=1, \ldots, m$. Defining the constrained Hamiltonian

$$
H_{c}(q, p, r):=H(q, p, r, s(q, p, r))
$$

it follows that (4.68) reduces to the same format as (2.7):

$$
\begin{aligned}
\dot{q} & =\frac{\partial H_{c}}{\partial p}(q, p, r), \\
\dot{p} & =-\frac{\partial H_{c}}{\partial q}(q, p, r), \\
\dot{r} & =0,
\end{aligned}
$$

which is an explicit Hamiltonian dynamics on the constrained state space $\mathcal{X}_{c}=$ $\left\{(q, p, r, s) \mid \frac{\partial H}{\partial s_{i}}(q, p, r, s)=0, i=1, \cdots, m\right\}$. Also note that while under the assumption (4.70) the variables $s_{1}, \ldots, s_{m}$ together with the Hamiltonian $H$ define a (constraint) submanifold $\mathcal{X}_{c}$ of $\mathcal{X}$, dually the level sets of the variables $r_{1}, \ldots, r_{\ell}$ define a foliation of $\mathcal{X}$. Both the constraint submanifold $\mathcal{X}_{c}$ and the foliation are invariant for the Hamiltonian dynamics. However, as shown in this section, there are cases of interest where the generalized Dirac structure does not satisfy the closedness condition (e.g., mechanical systems with nonholonomic constraints). Furthermore, also if the closedness condition is satisfied the actual construction of the canonical coordinates $q_{i}, p_{i}, r_{i}, s_{i}$, may be very involved, and preferably should be avoided.

We remark that the representation (4.68) of an implicit Hamiltonian system with regard to a closed Dirac structure is quite amenable for stability analysis, at least 
when the nondegeneracy condition (4.70) is satisfied. Indeed, let $\left(q_{0}, p_{0}, r_{0}, s_{0}\right)$ be an equilibrium of (4.68), that is,

$$
\frac{\partial H}{\partial q}\left(q_{0}, p_{0}, r_{0}, s_{0}\right)=0, \frac{\partial H}{\partial p}\left(q_{0}, p_{0}, r_{0}, s_{0}\right)=0, \frac{\partial H}{\partial s}\left(q_{0}, p_{0}, r_{0}, s_{0}\right)=0,
$$

and let us also assume that $\frac{\partial H}{\partial r}\left(q_{0}, p_{0}, r_{0}, s_{0}\right)=0$ (see later). Under the nondegeneracy condition (4.70) the implicit function theorem allows us to express the variables $s$ locally around $q_{0}, p_{0}, r_{0}, s_{0}$ as functions of $q, p, r$ leading as above to the explicit Hamiltonian dynamics (4.72). Note that in general the implicit function theorem only provides an existence result, and that finding the actual expression of $s$ as function of $q, p, r$ is in general not possible or preferably should be avoided.

Now, if the Hessian matrix of $H_{c}$ at $\left(q_{0}, p_{0}, r_{0}\right)$ is positive (or negative) definite it follows that $\left(q_{0}, p_{0}, r_{0}\right)$ is a stable equilibrium of (4.72) (see, e.g., $\left.[\mathrm{MR}]\right)$. On the other hand, this Hessian matrix of $H_{c}$ can be easily expressed in the original Hamiltonian $H$ as

$$
\left[\begin{array}{lll}
\frac{\partial^{2} H}{\partial q^{2}} & \frac{\partial^{2} H}{\partial q \partial p} & \frac{\partial^{2} H}{\partial q \partial r} \\
\frac{\partial^{2} H}{\partial p \partial q} & \frac{\partial^{2} H}{\partial p^{2}} & \frac{\partial^{2} H}{\partial p \partial r} \\
\frac{\partial^{2} H}{\partial r \partial q} & \frac{\partial^{2} H}{\partial r \partial p} & \frac{\partial^{2} H}{\partial r^{2}}
\end{array}\right]-\left[\begin{array}{c}
\frac{\partial^{2} H}{\partial q \partial s} \\
\frac{\partial^{2} H}{\partial p \partial s} \\
\frac{\partial^{2} H}{\partial r \partial s}
\end{array}\right]\left[\frac{\partial^{2} H}{\partial s^{2}}\right]^{-1}\left[\begin{array}{lll}
\frac{\partial^{2} H}{\partial s \partial q} & \frac{\partial^{2} H}{\partial s \partial p} & \frac{\partial^{2} H}{\partial s \partial r}
\end{array}\right]
$$

evaluated at $\left(q_{0}, p_{0}, r_{0}, s_{0}\right)$. Thus this way of checking stability can be performed without the actual computation of $H_{c}$. Furthermore, note that for checking definiteness of (4.74) only the variables $s$ need to be explicitly computed; we may use other coordinates instead of $q, p, r$.

Since the variables $r_{1}, \ldots, r_{l}$ are invariants (or Casimirs) we may also replace in the stability analysis the constrained Hamiltonian $H_{c}$ by $H_{c}(q, p, r)+\Phi(r)$, with $\Phi$ any function of $r=\left(r_{1}, \ldots, r_{l}\right)$. Hence we may also replace $H(q, p, r, s)$ with

$$
\bar{H}_{\Phi}(q, p, r, s):=H(q, p, r, s)+\Phi(r)
$$

and substitute $\bar{H}_{\Phi}$ into (4.74) in order to check definiteness. (The addition of a function $\Phi(r)$ to $H_{c}$ when checking the definiteness of the Hessian is known as the energy-Casimir method; see, e.g., [MR].)

5. Implicit port-controlled generalized Hamiltonian systems. As already alluded to in section 2, if we interconnect port-controlled Hamiltonian systems (2.1) in such a way that some of the external variables remain free port variables, then we will end up with an implicit generalized Hamiltonian system with external (or port) variables. In order to make this precise we give the following definition (see [SM2]).

DeFINITION 5.1. Let $\mathcal{X}$ be an $n$-dimensional manifold of energy variables, and let $H: \mathcal{X} \rightarrow \mathbb{R}$ be a Hamiltonian. Furthermore, let $\mathcal{F}$ be the linear space $\mathbb{R}^{m}$ of external flows $f$, with dual the space $\mathcal{F}^{*}$ of external efforts e. Consider a Dirac structure on the product space $\mathcal{X} \times \mathcal{F}$, only depending on $x$. The implicit port-controlled generalized Hamiltonian system corresponding to $\mathcal{X}, H, \mathcal{D}$, and $\mathcal{F}$ is defined by the specification

$$
\left(\dot{x}, f, \frac{\partial H}{\partial x}(x),-e\right) \in \mathcal{D}(x) .
$$

REMARK 5.1. The minus sign in front of the effort e comes from the natural identification $(\alpha, e) \in T^{*} \mathcal{X} \times \mathcal{F}^{*} \rightarrow(\alpha,-e) \in(T \mathcal{X} \times \mathcal{F})^{*}$. Physically this means that the ingoing power is counted positively. 
Since by definition of a Dirac structure (cf. (2.9)) $\langle\alpha \mid X\rangle-\langle e \mid f\rangle=0$ for all $(X, f, \alpha,-e) \in \mathcal{D}$, it follows that an implicit port-controlled Hamiltonian system satisfies the energy balance

$$
\frac{d H}{d t}=e^{T} f
$$

Definition 5.1 generalizes the notion of an (explicit) port-controlled generalized Hamiltonian system (2.1) by noting that in this case the Dirac structure $\mathcal{D}$ on $\mathcal{X} \times \mathcal{F}$ is given by the specification $(X, f, \alpha,-e) \in \mathcal{D}$ iff

$$
\begin{aligned}
& X(x)=J(x) \alpha(x)+g(x) f, \\
& e \quad=g^{T}(x) \alpha(x), \quad x \in \mathcal{X} .
\end{aligned}
$$

Indeed, let $(X, f, \alpha,-e) \in \mathcal{D}^{\perp}$; that is,

$$
\langle\hat{\alpha} \mid X\rangle+\langle\alpha \mid \hat{X}\rangle-\langle\hat{e} \mid f\rangle-\langle e \mid \hat{f}\rangle=0
$$

for all $(\hat{X}, \hat{f}, \hat{\alpha},-\hat{e})$ satisfying (5.3). By first taking $\hat{f}=0$ we obtain

$$
\hat{\alpha}^{T}(x) X(x)+\alpha^{T}(x) J(x) \hat{\alpha}(x)-\hat{\alpha}^{T}(x) g(x) f=0
$$

for all $\hat{\alpha}$, and thus $X(x)=J(x) \alpha(x)+g(x) f$, and substitution in (5.4) yields

$$
\hat{\alpha}^{T}(x) g(x) f+\alpha^{T}(x) g(x) \hat{f}-\hat{\alpha}^{T}(x) g(x) f-e^{T} \hat{f}=0
$$

for all $\hat{f}$, implying that $e=g^{T}(x) \alpha(x)$, and thus that $(X, f, \alpha,-e) \in \mathcal{D}$.

Now let us consider, as in section $2, k$ port-controlled generalized Hamiltonian systems, see $(2.15)$, with $\mathcal{E}_{j}=\mathcal{F}_{j}^{*}, j=1, \ldots, k$. A power-conserving partial interconnection is obtained by writing a direct sum decomposition

$$
\mathcal{F}_{1} \times \cdots \times \mathcal{F}_{k}=\mathcal{F}^{i} \oplus \mathcal{F}^{p}
$$

with the subspace $\mathcal{F}^{i}$ denoting the flows to be interconnected, and $\mathcal{F}^{p}$ the remaining flows at the external ports of the partially interconnected system. By defining $\mathcal{E}^{i}:=$ $\left(\mathcal{F}^{p}\right)^{\perp}$ and $\mathcal{E}^{p}:=\left(\mathcal{F}^{i}\right)^{\perp}$ we obtain the dual direct sum decomposition

$$
\mathcal{E}_{1} \times \cdots \times \mathcal{E}_{k}=\mathcal{E}^{i} \oplus \mathcal{E}^{p} .
$$

Proposition 5.1. Consider as in (2.15) $k$ port-controlled generalized Hamiltonian systems, with direct sum decomposition (5.7), (5.8). Consider a power-conserving partial interconnection given by a subspace (possibly parametrized by $x_{1}, \ldots, x_{k}$ )

$$
I\left(x_{1}, \ldots, x_{k}\right) \subset \mathcal{F}^{i} \times \mathcal{E}^{i}
$$

with $\operatorname{dim} I\left(x_{1}, \ldots, x_{k}\right)=\operatorname{dim} \mathcal{F}^{\mathrm{i}}$, having the property

$$
\left(f^{i}, e^{i}\right) \in I\left(x_{1}, \ldots, x_{k}\right) \Rightarrow\left\langle e^{i} \mid f^{i}\right\rangle=0 .
$$

Then the resulting partially interconnected system is an implicit port-controlled generalized Hamiltonian system with state space $\mathcal{X}:=\mathcal{X}_{1} \times \cdots \times \mathcal{X}_{k}$, Hamiltonian 
$H\left(x_{1}, \ldots, x_{k}\right):=H_{1}\left(x_{1}\right)+\cdots+H_{k}\left(x_{k}\right)$, and generalized Dirac structure on $\mathcal{X} \times \mathcal{F}^{p}$ given as

$$
\begin{aligned}
& \left(X, f^{p}, \alpha,-e^{p}\right)=\left(X_{1}, \ldots, X_{k}, f^{p}, \alpha_{1}, \ldots, \alpha_{k},-e^{p}\right) \in \mathcal{D} \Longleftrightarrow \\
& X_{j}\left(x_{j}\right)=J_{j}\left(x_{j}\right) \alpha_{j}\left(x_{j}\right)+g_{j}\left(x_{j}\right) f_{j}, \\
& e_{j}=g_{j}^{T}\left(x_{j}\right) \alpha_{j}\left(x_{j}\right), \quad x_{j} \in \mathcal{X}_{j}, \quad j=1, \ldots, k, \\
& \left(f_{1}, \ldots, f_{k}, e_{1}, \ldots, e_{k}\right)=\left(f^{i}, f^{p}, e^{i}, e^{p}\right) \text { such that }\left(f^{i}, e^{i}\right) \in I\left(x_{1}, \ldots, x_{k}\right) .
\end{aligned}
$$

Proof. The proof is very similar to the proof of Proposition 2.2. Let $\left(X, f^{p}, \alpha,-e^{p}\right)$ be in $\mathcal{D}^{\perp}$, that is,

$$
\langle\hat{\alpha} \mid X\rangle+\langle\alpha \mid \hat{X}\rangle-\left\langle\hat{e}^{p} \mid f^{p}\right\rangle-\left\langle e^{p} \mid \hat{f}^{p}\right\rangle=0
$$

for all $\left(\hat{X}, \hat{f}^{p}, \hat{\alpha},-\hat{e}^{p}\right)$ satisfying (5.11). First, letting $\hat{f}_{j}=\hat{e}_{j}=0$ for all $j=1, \ldots, k$ we obtain (2.21), and by substitution in (5.12) we obtain, similar to (2.22),

$$
0=\sum_{j=1}^{k}\left(\hat{e}_{j}^{T} f_{j}+e_{j}^{T} \hat{f}_{j}\right)-\left\langle\hat{e}^{p} \mid f^{p}\right\rangle-\left\langle e^{p} \mid \hat{f}^{p}\right\rangle=\left\langle\hat{e}^{i} \mid f^{i}\right\rangle+\left\langle e^{i} \mid \hat{f}^{i}\right\rangle
$$

for all $\hat{f}^{i}, \hat{e}^{i}$. By definition of $I\left(x_{1}, \ldots, x_{k}\right)$ in (5.10) this implies, as in Proposition 2.2 (adding if necessary flow vectors in the kernel of $\left.g_{j}\left(x_{i}\right)\right)$ that $\left(f^{i}, e^{i}\right) \in$ $I\left(x_{1}, \ldots, x_{k}\right)$, and thus $\left(X, f^{p}, \alpha,-e^{p}\right) \in \mathcal{D}$. Since it is readily seen that $\mathcal{D} \subset \mathcal{D}^{\perp}$ it follows that $\mathcal{D}$ defines a Dirac structure.

REMARK 5.2. An interesting open problem is the variational interpretation of Proposition 5.1 (and Proposition 2.2). Indeed, if all the Hamiltonian subsystems admit a variational characterization (as Euler-Lagrange equations) one could conjecture that also the (partially) interconnected Hamiltonian system admits "some kind of" variational characterization. It is to be expected, however, that the closedness conditions as treated in this and the previous section will play an important role in such a characterization, since already for classical mechanical systems with kinematic constraints it is known (see e.g., [AKN, BC]) that they cannot be formulated as standard Euler-Lagrange equations in case the constraints are nonholonomic. Also, the formulation (4.68) of an implicit Hamiltonian system satisfying the closedness condition suggests a connection with variational principles via the first-order condition of Pontryagin's maximum principle. In the case of electrical circuits, where the interconnections are defined by Kirchhoff's laws and the closedness conditions are trivially satisfied (see Example 3.1), some important work concerning a variational formulation of Kirchhoff's laws and the resulting variational characterization of the overall circuit has been done (see, e.g., [JE, M1]), and it seems of interest to extend these ideas to the general situation considered in Proposition 5.1.

In the rest of this section we will not elaborate on general implicit port-controlled Hamiltonian systems and their different representations, but instead concentrate on a special subclass which arises naturally in the control of mechanical systems. Consider the following port-controlled generalized Hamiltonian system with constraints given 
by

$$
\begin{aligned}
\dot{x} & =J(x) \frac{\partial H}{\partial x}(x)+g(x) f+b(x) \lambda \\
e & =g^{T}(x) \frac{\partial H}{\partial x}(x) \\
0 & =b^{T}(x) \frac{\partial H}{\partial x}(x)
\end{aligned}
$$

where $x \in \mathcal{X}, f \in \mathcal{F}:=\mathbb{R}^{m}$ and $g(x)=\left[g_{1}(x) \ldots g_{m}(x)\right]$ is the $n \times m$ matrix of input vector fields $g_{j} . b(x)=\left[b_{1}(x) \ldots b_{k}(x)\right]$ is the $n \times k$ matrix of constraint vector fields. Throughout this section we will assume that $b(x)$ has rank equal to $k$ everywhere. It is easily seen that, e.g., an actuated mechanical system with kinematic constraints will fit into the description (5.14). By rewriting (5.14) as

$$
\begin{aligned}
{\left[\begin{array}{l}
\dot{x} \\
f
\end{array}\right] } & =\underbrace{\left[\begin{array}{cc}
J(x) & 0 \\
0 & 0
\end{array}\right]}_{\tilde{J}(x)}\left[\begin{array}{c}
\frac{\partial H}{\partial x}(x) \\
-e
\end{array}\right]+\left[\begin{array}{cc}
g(x) & b(x) \\
I_{m} & 0
\end{array}\right] \tilde{\lambda}, \\
0 & =\left[\begin{array}{cc}
g^{T}(x) & I_{m} \\
b^{T}(x) & 0
\end{array}\right]\left[\begin{array}{c}
\frac{\partial H}{\partial x}(x) \\
-e
\end{array}\right],
\end{aligned}
$$

where $\tilde{\lambda} \in \mathbb{R}^{m+k}$, it follows from Theorem 3.1 that (5.15) defines representation II of a generalized Dirac structure $\mathcal{D}$ on $\mathcal{X} \times \mathcal{F}$. Thus (5.14) is an implicit port-controlled generalized Hamiltonian system.

We will now study $\mathcal{D}$ further as given in representation (5.15). In what follows we will use $\{$,$\} and \{,\}_{\mathcal{X} \times \mathcal{F}}$ to denote the generalized Poisson brackets on $\mathcal{X}$ and $\mathcal{X} \times \mathcal{F}$, respectively, with structure matrices $J(x)$ and $\tilde{J}(x)$ (see $(5.15)$ ), respectively. In addition we will let $B$ denote the constant-dimensional distribution on $\mathcal{X}$ given by

$$
B(x)=\operatorname{Im} b(x), \quad x \in \mathcal{X} .
$$

From (5.15) we immediately see that the distribution $G_{0}$ on $\mathcal{X} \times \mathcal{F}$ defined by $\mathcal{D}$ (see $(3.1)$ ) is given by

$$
G_{0}(x, y)=\operatorname{Im}\left[\begin{array}{cc}
g(x) & b(x) \\
I_{m} & 0
\end{array}\right], \quad(x, y) \in \mathcal{X} \times \mathcal{F} .
$$

Note that $G_{0}$ is constant-dimensional with dimension equal to $m+k$ since $\operatorname{rank} b(x)=$ $k$ for all $x \in \mathcal{X}$. The following lemma, for which a proof is straightforward, gives necessary and sufficient conditions for $G_{0}$ being involutive.

LEMma 5.1. $G_{0}$ is involutive if and only if $[X, Y] \in B$ for all $X, Y$ $\in\left\{g_{1}, \ldots, g_{m}, b_{1}, \ldots, b_{k}\right\}$.

The next lemma gives three necessary conditions for the closedness of $\mathcal{D}$.

Lemma 5.2. If the generalized Dirac structure $\mathcal{D}$ on $\mathcal{X} \times \mathcal{F}$ is closed, then

1. $\left\{H_{1},\left\{H_{2}, H_{3}\right\}\right\}+\left\{H_{2},\left\{H_{3}, H_{1}\right\}\right\}+\left\{H_{3},\left\{H_{1}, H_{2}\right\}\right\}=0$;

2. $L_{g_{j}}\left\{H_{1}, H_{2}\right\}=\left\{L_{g_{j}} H_{1}, H_{2}\right\}+\left\{H_{1}, L_{g_{j}} H_{2}\right\}, j=1, \ldots, m$;

3. $\mathbf{d}\left\{H_{1}, H_{2}\right\} \in \operatorname{ann} B$

for all $H_{1}, H_{2}, H_{3} \in C^{\infty}(\mathcal{X})$ such that $\mathbf{d} H_{1}, \mathbf{d} H_{2}, \mathbf{d} H_{3} \in \operatorname{ann} B$.

Proof. Assume that $\mathcal{D}$ is closed, i.e., satisfies (4.1). Using Cartan's magic formula, the closedness condition (4.1) can be written as

$$
\begin{aligned}
\left\langle\mathbf{d}\left\langle\alpha_{2} \mid X_{1}\right\rangle \mid X_{3}\right\rangle+\left\langle\mathbf{d}\left\langle\alpha_{3} \mid X_{2}\right\rangle \mid X_{1}\right\rangle+\left\langle\mathbf{d}\left\langle\alpha_{1} \mid X_{3}\right\rangle \mid X_{2}\right\rangle & \\
+ & \mathbf{d} \alpha_{2}\left(X_{1}, X_{3}\right)+\mathbf{d} \alpha_{3}\left(X_{2}, X_{1}\right)+\mathbf{d} \alpha_{1}\left(X_{3}, X_{2}\right)=0 .
\end{aligned}
$$


Consider $H_{1}, H_{2}$, and $H_{3} \in C^{\infty}(\mathcal{X})$, where $\mathbf{d} H_{i} \in$ ann $B, i=1,2,3$. Let $j \in$ $\{1, \ldots, m\}$ and define

$$
\begin{array}{ll}
X_{1}(x, y)=\left[\begin{array}{c}
X_{H_{1}}(x) \\
0
\end{array}\right], & \alpha_{1}(x, y)=\left[\begin{array}{c}
\frac{\partial H_{1}}{\partial x}(x) \\
-L_{g}^{T} H_{1}(x)
\end{array}\right], \\
X_{2}(x, y)=\left[\begin{array}{c}
X_{H_{2}}(x) \\
0
\end{array}\right], & \alpha_{2}(x, y)=\left[\begin{array}{c}
\frac{\partial H_{2}}{\partial x}(x) \\
-L_{g}^{T} H_{2}(x)
\end{array}\right], \\
X_{3}(x, y)=\left[\begin{array}{c}
X_{H_{3}}(x)+\rho g_{j}(x) \\
\rho Y_{j}
\end{array}\right], & \alpha_{3}(x, y)=\left[\begin{array}{c}
\frac{\partial H_{3}}{\partial x}(x) \\
-L_{g}^{T} H_{3}(x)
\end{array}\right]
\end{array}
$$

for $(x, y) \in \mathcal{X} \times \mathcal{F}$, where $Y_{j}=\frac{\partial}{\partial y_{j}}, \rho \in \mathbb{R}$ and

$$
X_{H_{i}}(x)=J(x) \frac{\partial H_{i}}{\partial x}(x), L_{g}^{T} H_{i}(x)=g^{T}(x) \frac{\partial H_{i}}{\partial x}(x) .
$$

Thus, $\left(X_{i}, \alpha_{i}\right) \in \mathcal{D}, i=1,2,3$. Now, it is easy to see that $\left\langle\alpha_{i} \mid X_{j}\right\rangle=\left\{H_{i}, H_{j}\right\}$, $i, j=1,2,3$, which implies that

$$
\begin{aligned}
& \left\langle\mathbf{d}\left\langle\alpha_{2} \mid X_{1}\right\rangle \mid X_{3}\right\rangle=\left\{\left\{H_{2}, H_{1}\right\}, H_{3}\right\}+\rho L_{g_{j}}\left\{H_{2}, H_{1}\right\}, \\
& \left\langle\mathbf{d}\left\langle\alpha_{3} \mid X_{2}\right\rangle \mid X_{1}\right\rangle=\left\{\left\{H_{3}, H_{2}\right\}, H_{1}\right\}, \\
& \left\langle\mathbf{d}\left\langle\alpha_{1} \mid X_{3}\right\rangle \mid X_{2}\right\rangle=\left\{\left\{H_{1}, H_{3}\right\}, H_{2}\right\} .
\end{aligned}
$$

Moreover, we have that

$$
\alpha_{i}=\frac{\partial H_{i}}{\partial x_{1}} \mathbf{d} x_{1}+\cdots+\frac{\partial H_{i}}{\partial x_{n}} \mathbf{d} x_{n}-L_{g_{1}} H_{i} \mathbf{d} y_{1}-\cdots-L_{g_{m}} H_{i} \mathbf{d} y_{m}, \quad i=1, \ldots, 3,
$$

which means that

$$
\mathbf{d} \alpha_{i}=-\sum_{l=1}^{m} \sum_{k=1}^{n} \frac{\partial L_{g_{l}} H_{i}}{\partial x_{k}} \mathbf{d} x_{k} \wedge \mathbf{d} y_{l}, \quad i=1, \ldots, 3 .
$$

Hence,

$$
\mathbf{d} \alpha_{2}\left(X_{1}, X_{3}\right)=-\rho\left[\frac{\partial L_{g_{j}} H_{2}}{\partial x}\right]^{T} X_{H_{1}}=-\rho\left\{L_{g_{j}} H_{2}, H_{1}\right\}
$$

and similarly

$$
\mathbf{d} \alpha_{1}\left(X_{3}, X_{2}\right)=-\mathbf{d} \alpha_{1}\left(X_{2}, X_{3}\right)=\rho\left\{L_{g_{j}} H_{1}, H_{2}\right\}=-\rho\left\{H_{2}, L_{g_{j}} H_{1}\right\} .
$$

In addition, it follows that $\mathbf{d} \alpha_{3}\left(X_{2}, X_{1}\right)=0$. Therefore, from the integrability condition (5.18) we have that

$$
\begin{aligned}
\left\{\left\{H_{2}, H_{1}\right\}, H_{3}\right\}+\left\{\left\{H_{3}, H_{2}\right\}, H_{1}\right\}+\left\{\left\{H_{1}, H_{3}\right\}, H_{2}\right\} \\
+\rho\left(L_{g_{j}}\left\{H_{2}, H_{1}\right\}-\left\{L_{g_{j}} H_{2}, H_{1}\right\}-\left\{H_{2}, L_{g_{j}} H_{1}\right\}\right)=0
\end{aligned}
$$

for all $\rho \in \mathbb{R}$, implying condition 1 for $\rho=0$ and condition 2 for $\rho=1$. 
Now, a direct calculation yields

$$
\begin{aligned}
\alpha_{12} & =\mathbf{i}_{X_{2}} \mathbf{d} \alpha_{1}-\mathbf{i}_{X_{1}} \mathbf{d} \alpha_{2}+\mathbf{d}\left\langle\alpha_{1} \mid X_{2}\right\rangle \\
& =-\sum_{l=1}^{m}\left(\left\{L_{g_{l}} H_{1}, H_{2}\right\}+\left\{H_{1}, L_{g_{l}} H_{2}\right\}\right) \mathbf{d} y_{l}+\sum_{k=1}^{n} \frac{\partial\left\{H_{1}, H_{2}\right\}}{\partial x_{k}} \mathbf{d} x_{k},
\end{aligned}
$$

from which condition 3 (and condition 2) follows directly since $\alpha_{12} \in$ ann $G_{0}$ (see Theorem 4.1).

Before being able to give the sufficient and necessary conditions for $\mathcal{D}$ being closed, we also need the following result.

LEMmA 5.3. If for arbitrary $H_{1}, H_{2} \in C^{\infty}(\mathcal{X})$ such that $\mathbf{d} H_{1}, \mathbf{d} H_{2} \in$ ann $B$ there holds

1. $L_{g_{j}}\left\{H_{1}, H_{2}\right\}=\left\{L_{g_{j}} H_{1}, H_{2}\right\}+\left\{H_{1}, L_{g_{j}} H_{2}\right\}, j=1, \ldots, m$,

2. $\mathbf{d}\left\{H_{1}, H_{2}\right\} \in$ ann $B$,

then $\left\{\tilde{H}_{1}, \tilde{H}_{2}\right\} \mathcal{X} \times \mathcal{F} \in \mathcal{A}_{\mathcal{D}}$ for all $\tilde{H}_{1}, \tilde{H}_{2} \in \mathcal{A}_{\mathcal{D}}$

Proof. Take arbitrary $\tilde{H}_{2}, \tilde{H}_{2} \in \mathcal{A}_{\mathcal{D}}$. From (5.17) we see that this is equivalent to

$$
\begin{aligned}
0 & =b^{T}(x) \frac{\partial \tilde{H}_{i}}{\partial x}(x, y), \\
\frac{\partial \tilde{H}_{i}}{\partial y}(x, y) & =-g^{T}(x) \frac{\partial \tilde{H}_{i}}{\partial x}(x, y)
\end{aligned}
$$

for $i=1,2$. Let $j \in\{1, \ldots, m\}$ and define

$$
\hat{g}_{j}(x, y)=\left[\begin{array}{c}
g_{j}(x) \\
0
\end{array}\right]
$$

Then (5.33) can be written as

$$
\frac{\partial \tilde{H}_{i}}{\partial y_{k}}=-L_{\hat{g}_{k}} \tilde{H}_{i}, \quad k=1, \ldots, m
$$

for $i=1,2$. Now,

$$
\left\{\tilde{H}_{1}, \tilde{H}_{2}\right\}_{\mathcal{X} \times \mathcal{F}}=\sum_{k, l=1}^{n} J_{k l}(x) \frac{\partial \tilde{H}_{1}}{\partial x_{k}} \frac{\partial \tilde{H}_{2}}{\partial x_{l}},
$$

so

$$
\frac{\partial\left\{\tilde{H}_{1}, \tilde{H}_{2}\right\}_{\mathcal{X} \times \mathcal{F}}}{\partial y_{j}}=-\left\{L_{\hat{g}_{j}} \tilde{H}_{1}, \tilde{H}_{2}\right\}_{\mathcal{X} \times \mathcal{F}}-\left\{\tilde{H}_{1}, L_{\hat{g}_{j}} \tilde{H}_{2}\right\}_{\mathcal{X} \times \mathcal{F}}
$$

Since

$$
\left\{H_{1}, H_{2}\right\}=\sum_{k, l=1}^{n} J_{k l}(x) \frac{\partial H_{1}}{\partial x_{k}} \frac{\partial H_{2}}{\partial x_{l}}, H_{1}, H_{2} \in C^{\infty}(\mathcal{X}),
$$

it follows from condition 1 that

$$
L_{\hat{g}_{j}}\left\{\tilde{H}_{1}, \tilde{H}_{2}\right\}_{\mathcal{X} \times \mathcal{F}}=\left\{L_{\hat{g}_{j}} \tilde{H}_{1}, \tilde{H}_{2}\right\}_{\mathcal{X} \times \mathcal{F}}+\left\{\tilde{H}_{1}, L_{\hat{g}_{j}} \tilde{H}_{2}\right\}_{\mathcal{X} \times \mathcal{F}},
$$


which inserted in (5.37) yields

$$
\frac{\partial\left\{\tilde{H}_{1}, \tilde{H}_{2}\right\}_{\mathcal{X} \times \mathcal{F}}}{\partial y_{j}}=-L_{\hat{g}_{j}}\left\{\tilde{H}_{1}, \tilde{H}_{2}\right\}_{\mathcal{X} \times \mathcal{F}} .
$$

Moreover, from condition 2 it follows that

$$
b^{T}(x) \frac{\partial\left\{\tilde{H}_{1}, \tilde{H}_{2}\right\}_{\mathcal{X} \times \mathcal{F}}}{\partial x}=0 .
$$

Thus, from (5.40) and (5.41) we see that $\left\{\tilde{H}_{1}, \tilde{H}_{2}\right\}_{\mathcal{X} \times \mathcal{F}} \in \mathcal{A}_{\mathcal{D}}$.

We are now ready to present the necessary and sufficient conditions for (5.15) defining a Dirac structure on $\mathcal{X} \times \mathcal{F}$.

TheOREM 5.1. The generalized Dirac structure $\mathcal{D}$ on $\mathcal{X} \times \mathcal{F}$ as defined by (5.15) is closed if and only if

1. $[X, Y] \in B$ for all vector fields $X, Y \in\left\{g_{1}, \ldots, g_{m}, b_{1}, \ldots, b_{k}\right\}$;

2. $L_{g_{j}}\left\{H_{1}, H_{2}\right\}=\left\{L_{g_{j}} H_{1}, H_{2}\right\}+\left\{H_{1}, L_{g_{j}} H_{2}\right\}, j=1, \ldots, m$;

3. $\mathbf{d}\left\{H_{1}, H_{2}\right\} \in$ ann $B$;

4. $\left\{H_{1},\left\{H_{2}, H_{3}\right\}\right\}+\left\{H_{2},\left\{H_{3}, H_{1}\right\}\right\}+\left\{H_{3},\left\{H_{1}, H_{2}\right\}\right\}=0$ for all $H_{1}, H_{2}, H_{3} \in C^{\infty}(\mathcal{X})$ such that $\mathbf{d} H_{1}, \mathbf{d} H_{2}, \mathbf{d} H_{3} \in$ ann $B$.

Proof. The necessary and sufficient conditions for closedness of $\mathcal{D}$ follows immediately by combining the results in Lemma 5.1, Lemma 5.2, Lemma 5.3 and then using Theorem 4.4.

Corollary $5.1(B=0)$. Let $b(x)=0$ (no constraints) in (5.14). Then the generalized Dirac structure $\mathcal{D}$ on $\mathcal{X} \times \mathcal{F}$ as defined by (5.15) (with $b(x)=0$ ) is closed if and only if

1. $\left[g_{i}, g_{j}\right]=0, i, j=1, \ldots, m$;

2. $L_{g_{j}}\left\{H_{1}, H_{2}\right\}=\left\{L_{g_{j}} H_{1}, H_{2}\right\}+\left\{H_{1}, L_{g_{j}} H_{2}\right\}$ for all $H_{1}, H_{2} \in C^{\infty}(\mathcal{X})$, $j=1, \ldots, m$;

3. $\{$,$\} satisfies the Jacobi identity.$

Hence, the closedness condition (4.1) for the generalized Dirac structure on $\mathcal{X} \times$ $\mathcal{F}$ arising from the constrained port-controlled Hamiltonian system (5.14) translates (among other things) into strong conditions on the input vector fields $g_{j}$.

Conditions 2-4 in Theorem 5.1 may be succinctly expressed by requiring that the generalized Poisson bracket $\{$,$\} of F, G \in C^{\infty}(\mathcal{X})$ where $\mathbf{d} F, \mathbf{d} G \in$ ann $B$ is preserved by the dynamics of (5.14) for every choice of internal energy $H$ such that $\mathbf{d} H \in$ ann $B$ and for every $f \in \mathcal{F}$. Indeed, requiring that

$$
\frac{d}{d t}\{F, G\}=\left\{\frac{d}{d t} F, G\right\}+\left\{F, \frac{d}{d t} G\right\}
$$

for all $F, G \in C^{\infty}(\mathcal{X})$ such that $\mathbf{d} F, \mathbf{d} G \in$ ann $B$, where $\frac{d}{d t}$ denotes the time-derivative along (5.14), is equivalent to

$$
\begin{aligned}
\{\{F, G\}, H\}+ & L_{g}\{F, G\} f+L_{b}\{F, G\} \lambda \\
& =\{\{F, H\}, G\}+\left\{\left(L_{g} F\right) f, G\right\}+\{F,\{G, H\}\}+\left\{F,\left(L_{g} G\right) f\right\}
\end{aligned}
$$

for all $H \in C^{\infty}(\mathcal{X})$ such that $\mathbf{d} H \in \operatorname{ann} B$ and $f \in \mathcal{F}$. Letting $H=0$ and $f=0$, we obtain

$$
L_{b}\{F, G\} \lambda=0 \text { for all } \lambda \in \mathbb{R}^{k}
$$


which means that $\mathbf{d}\{F, G\} \in \operatorname{ann} B$. Moreover, letting $f=0$ leads to

$$
\{\{F, G\}, H\}=\{\{F, H\}, G\}+\{F,\{G, H\}\},
$$

which is none other than the Jacobi-identity. Thus, (5.43) amounts to

$$
L_{g}\{F, G\} f=\left\{\left(L_{g} F\right) f, G\right\}+\left\{F,\left(L_{g} G\right) f\right\} \quad \text { for all } f \in \mathcal{F},
$$

which is equivalent to

$$
L_{g_{j}}\{F, G\}=\left\{L_{g_{j}} F, G\right\}+\left\{F, L_{g_{j}} G\right\}, \quad j=1, \ldots, m .
$$

The next example should give an idea of what the conditions in Theorem 5.1 imply for the (local) mathematical structure of system (5.14).

EXAMPLE 5.1. Consider the port-controlled generalized Hamiltonian system with constraints given in (5.14). Assume that conditions 1-4 in Theorem 5.1 are all satisfied. By condition 1 it follows that the constant-dimensional distribution $B$ is involutive. Hence, by Frobenius' theorem in a neighborhood of any point $x_{0} \in \mathcal{X}$ there exist local coordinates $(y, s)=\left(y_{1}, \ldots, y_{n-k}, s_{1}, \ldots, s_{k}\right)$, such that

$$
\operatorname{ann} B=\operatorname{span}\left\{\mathbf{d} y_{1}, \ldots, \mathbf{d} y_{n-k}\right\}
$$

and

$$
B=\operatorname{span}\left\{\frac{\partial}{\partial s_{1}}, \ldots, \frac{\partial}{\partial s_{k}}\right\}
$$

Condition 3 implies that

$$
\frac{\partial\left\{y_{i}, y_{j}\right\}}{\partial s_{l}}=0, \quad l=1, \ldots, k, i, j=1, \ldots, n-k .
$$

Hence, $J(x)$ takes the following form in the local coordinates $(y, s)$ :

$$
J(y, s)=\left[\begin{array}{cc}
J_{y y}(y) & * \\
* & *
\end{array}\right]
$$

where $J_{y y}(y)=\left[\left\{y_{i}, y_{j}\right\}\right]$ is the $(n-k) \times(n-k)$ upper-left submatrix of $J(y, s)$. From condition 1 it also follows that $\left[b_{i}, g_{j}\right] \in B$ which implies that in the coordinates $(y, s)$ the matrix of input vector fields takes the form

$$
g(x, y)=\left[\begin{array}{c}
g_{y}(y) \\
g_{s}(y, s)
\end{array}\right] .
$$

Furthermore, since

$$
\left[g_{i}, g_{j}\right](y, s)=\left[\begin{array}{c}
{\left[g_{y_{i}}, g_{y_{j}}\right](y)} \\
*
\end{array}\right]
$$

while $\left[g_{i}, g_{j}\right] \in B$, it follows that $\left[g_{y_{i}}, g_{y_{j}}\right]=0, i, j=1, \ldots, m$. Assume additionally that the distribution $B+G$ is constant-dimensional with dimension equal to $m+k$. Then the submatrix $g_{y}(y)$ of $g(x, y)$ has constant rank equal to $m \leq n-k$. Thus 
(see e.g., Theorem 2.36 in $[\mathrm{NS}])$, there exists a local transformation $\left(y_{1}, \ldots, y_{n-k}\right) \rightarrow$ $\left(\tilde{y}_{1}, \ldots, \tilde{y}_{n-k}\right)$ such that

$$
g_{y_{j}}=\frac{\partial}{\partial \tilde{y}_{j}}, \quad j=1, \ldots m
$$

In these coordinates condition 2 amounts to

$$
\frac{\partial\left\{\tilde{y}_{i}, \tilde{y}_{j}\right\}}{\partial \tilde{y}_{l}}=\left\{\frac{\partial \tilde{y}_{i}}{\partial \tilde{y}_{l}}, \tilde{y}_{j}\right\}+\left\{\tilde{y}_{i}, \frac{\partial \tilde{y}_{j}}{\partial \tilde{y}_{l}}\right\}=0, \quad l=1, \ldots, m, i, j=1, \ldots n-k,
$$

which means that $\left\{\tilde{y}_{i}, \tilde{y}_{j}\right\}$ is independent of the first $m$ local coordinates $\tilde{y}_{1}, \ldots, \tilde{y}_{m}$. Let now $z=\left(\tilde{y}_{m+1}, \ldots, \tilde{y}_{n-k}\right)$ and $w=\left(\tilde{y}_{1}, \ldots, \tilde{y}_{m}\right)$. Then from the discussion above we can conclude that $(z, w, s)$ are local coordinates for $\mathcal{X}$ around $x_{0}$ in which (5.14) takes the form

$$
\begin{aligned}
{\left[\begin{array}{c}
\dot{z} \\
\dot{w}
\end{array}\right]=} & {\left[\begin{array}{cc}
J_{z z}(z) & J_{z w}(z) \\
-J_{z w}^{T}(z) & J_{w w}(z)
\end{array}\right]\left[\begin{array}{c}
\frac{\partial H}{\partial z}(z, w, s) \\
\frac{\partial H}{\partial w}(z, w, s)
\end{array}\right]+\left[\begin{array}{c}
0 \\
I_{m}
\end{array}\right] f } \\
\dot{s}= & J_{s z}(z, w, s) \frac{\partial H}{\partial z}(z, w, s)+J_{s w}(z, w, s) \frac{\partial H}{\partial w}(z, w, s) \\
& +g_{s}(z, w, s) f+b_{s}(z, w, s) \lambda \\
e= & \frac{\partial H}{\partial w}(z, w, s) \\
0= & \frac{\partial H}{\partial s}(z, w, s)
\end{aligned}
$$

where the last equation follows from the fact that the $k \times k$ matrix $b_{s}(z, w, s)$ has full rank. Note that the equation for $\dot{s}$ can be left out from (5.56) because it is needed only to determine the Lagrange multipliers $\lambda \in \mathbb{R}^{k}$. Finally from condition 4 it follows that the matrix

$$
\left[\begin{array}{cc}
J_{z z}(z) & J_{z w}(z) \\
-J_{z w}^{T}(z) & J_{w w}(z)
\end{array}\right]
$$

satisfies the Jacobi-identity (in the (z,w)-coordinates).

Finally, in the next example we will relate the results in this paper (in particular this section) to "passivity-based control" of actuated mechanical systems with kinematic constraints.

EXAMPLE 5.2. Consider a mechanical system with kinematic constraints $A^{T}(q) \dot{q}$ $=0$ as in Example 3.2. Additionally, let the system be actuated by generalized external forces $u=\left(u_{1}, \ldots, u_{m}\right)$ corresponding to generalized configuration coordinates $C_{1}(q), \ldots, C_{m}(q)$. The dynamical equations of motion are given as

$$
\begin{aligned}
& {\left[\begin{array}{c}
\dot{q} \\
\dot{p}
\end{array}\right]=\left[\begin{array}{cc}
0 & I_{n} \\
-I_{n} & 0
\end{array}\right]\left[\begin{array}{c}
\frac{\partial H}{\partial q}(q, p) \\
\frac{\partial H}{\partial p}(q, p)
\end{array}\right]+\sum_{i=1}^{m}\left[\begin{array}{c}
0 \\
\frac{\partial C_{i}}{\partial q}(q)
\end{array}\right] u_{i}+\left[\begin{array}{c}
0 \\
A(q)
\end{array}\right] \lambda,} \\
& y_{i} \quad=\left[\frac{\partial C_{i}}{\partial q}(q)\right]^{T} \frac{\partial H}{\partial p}(q, p)\left(=\frac{d C_{i}}{d t}(q)\right), \quad i=1, \ldots, m, \\
& 0 \quad=A^{T}(q) \frac{\partial H}{\partial p}(q, p)\left(=A^{T}(q) \dot{q}\right) .
\end{aligned}
$$


This is a port-controlled generalized Hamiltonian system with constraints as in (5.14) with external flows the vector $\left(u_{1}, \ldots, u_{m}\right)$ of external forces, and external efforts the vector $\left(y_{1}, \ldots, y_{m}\right)$ of corresponding generalized velocities. It can be verified, as in Proposition 4.1, that the underlying generalized Dirac structure satisfies the conditions of Theorem 5.1 (that is, is closed) if and only if the kinematic constraints $A^{T}(q) \dot{q}=0$ are holonomic.

Now, consider an additional port-controlled Hamiltonian system (the "controller")

$$
\begin{aligned}
\dot{\xi} & =u_{c}, \\
y_{c} & =\frac{\partial P}{\partial \xi}(\xi), \quad \xi, u_{c}, y_{c} \in \mathbb{R}^{m},
\end{aligned}
$$

with Hamiltonian $P$. (Note that this is of the type (2.1) with $J=0, g=$ identity matrix, $x=\xi, f=u_{c}$, and $e=y_{c}$.) Feedback interconnection as in Example 2.1 leads to the implicit generalized Hamiltonian system

$$
\begin{aligned}
{\left[\begin{array}{c}
\dot{q} \\
\dot{p} \\
\dot{\xi}
\end{array}\right] } & =\left[\begin{array}{ccc}
0 & I_{n} & 0 \\
-I_{n} & 0 & -\frac{\partial C}{\partial q}(q) \\
0 & \frac{\partial^{T} C}{\partial q}(q) & 0
\end{array}\right]\left[\begin{array}{c}
\frac{\partial H}{\partial q}(q, p) \\
\frac{\partial H}{\partial p}(q, p) \\
\frac{\partial P}{\partial \xi}(\xi)
\end{array}\right]+\left[\begin{array}{c}
0 \\
A(q) \\
0
\end{array}\right] \lambda, \\
0 & =A^{T}(q) \frac{\partial H}{\partial p}(q, p),
\end{aligned}
$$

with $\frac{\partial C}{\partial q}(q)$ denoting the matrix with ith column $\frac{\partial C_{i}}{\partial q}(q)$. The codistribution $P_{0}$ of the underlying generalized Dirac structure can be readily seen to be given as

$$
P_{0}=\operatorname{span}\left\{\mathbf{d} C_{i}-\mathbf{d} \xi_{i} \mid i=1, \ldots, m\right\}
$$

expressing the fact (see also Remark 3.1) that the functions

$$
C_{i}(q)-\xi_{i}, \quad i=1, \ldots, m,
$$

are independent conserved quantities for the closed-loop dynamics (5.60). It follows that along (5.60)

$$
\xi_{i}(t)=C_{i}(q(t))+c_{i}, \quad \text { for all } t, \quad i=1, \ldots, m,
$$

with the constants $c_{i}$ solely depending on the initial conditions of the "controller" (5.59).

Substituting (5.63) into (5.60) and noting that

$$
\frac{\partial C}{\partial q}(q) \frac{\partial P}{\partial \xi}\left(C_{1}(q)+c_{1}, \ldots, C_{m}(q)+c_{m}\right)=\frac{\partial P}{\partial q}\left(C_{1}(q)+c_{1}, \ldots, C_{m}(q)+c_{m}\right),
$$

it follows that the dynamics of the ( $q, p)$-part of (5.60) (the original mechanical system) are given as

$$
\begin{aligned}
& {\left[\begin{array}{c}
\dot{q} \\
\dot{p}
\end{array}\right]=\left[\begin{array}{cc}
0 & I_{n} \\
-I_{n} & 0
\end{array}\right]\left[\begin{array}{c}
\frac{\partial H_{\text {new }}}{\partial q}(q, p) \\
\frac{\partial H_{\text {new }}}{\partial p}(q, p)
\end{array}\right]+\left[\begin{array}{c}
0 \\
A(q)
\end{array}\right] \lambda,} \\
& 0 \quad=A^{T}(q) \frac{\partial H_{n e w}}{\partial p}(q, p),
\end{aligned}
$$


where $H_{\text {new }}$ is the "new" Hamiltonian defined by

$$
H_{\text {new }}(q, p)=H(q, p)+P\left(C_{1}(q)+c_{1}, \ldots, C_{m}(q)+c_{m}\right) .
$$

Thus by appropriately choosing the Hamiltonian $P(\xi)$ of the "controller sub-system" (5.59), we may shape the Hamiltonian $H(q, p)$ of the constrained mechanical system (5.58) by addition of the potential energy $P\left(C_{1}(q)+c_{1}, \ldots, C_{m}(q)+c_{m}\right)$, with $c_{1}, \ldots, c_{m}$ only depending on the initial condition of (5.59) (that is, with properly initialization we may set $\left.c_{1}=\ldots=c_{m}=0\right)$. This idea of shaping the internal energy is one of the main ideas of "passivity-based control." We have thus demonstrated that this can be accomplished by power-conserving (in fact, feedback) interconnection of (5.58) with a controller sub-system (5.59).

In particular, if $H$ and $C_{1}, \ldots, C_{m}$ are such that $P$ can be chosen in such a manner that $H_{\text {new }}$ as defined by (5.66) has a strict minimum at some desired equilibrium point $\left(q_{0}, p_{0}\right)$, then $\left(q_{0}, p_{0}\right)$ will be a (Lyapunov) stable equilibrium of $(5.65)$ (and, because of (5.63), also the $\xi$-dynamics will be stable). To be more precise we only need the function $H_{\text {new }}$ restricted to the constraint manifold $\left\{(q, p) \mid A^{T}(q) \frac{\partial H_{\text {new }}}{\partial p}(q, p)=0\right\}$ to have a strict minimum at $\left(q_{0}, p_{0}\right)$.

It can be verified that the underlying generalized Dirac structure of (5.60) is closed if and only if the kinematic constraints $A^{T}(q) \dot{q}=0$ are holonomic. If this happens to be the case then checking that $H_{\text {new }}$ restricted to the constraint manifold has a strict minimum may be performed as indicated at the end of section 4.

Within the same philosophy one may pursue asymptotic stability by adding, apart from the energy-shaping Hamiltonian controller (2.24), energy-dissipating elements to the system. In particular, one may replace the feedback interconnection $u_{c}=y$, $u=-y_{c}$ as above by the power-conserving partial interconnection (with free external flow $v$ and external effort $y$ )

$$
\begin{aligned}
& u_{c}=y, \\
& u=-y_{c}+v,
\end{aligned}
$$

and then terminate this port by an energy-dissipating element

$$
v=-\frac{\partial R}{\partial y}(y)
$$

for some (Rayleigh) dissipation function $R$. For the asymptotic stability analysis of the resulting closed-loop system one again must distinguish between holonomic and nonholonomic kinematic constraints $A^{T}(q) \dot{q}=0$. (In fact, in the nonholonomic case there is a fundamental obstruction to asymptotic stabilization, since Brockett's necessary conditions are not satisfied; see, e.g., [MS3] for the references.)

6. Conclusions. It has been shown that a power-conserving interconnection of port-controlled generalized Hamiltonian systems leads to an implicit generalized Hamiltonian system, and a power-conserving partial interconnection to an implicit port-controlled Hamiltonian system. The crucial concept is the notion of a (generalized) Dirac structure, defined on the space of energy-variables or on the product of the space of energy-variables and the space of flow-variables in the port-controlled case. Three natural representations of generalized Dirac structures have been treated. Necessary and sufficient conditions for closedness of a Dirac structure in all three representations have been obtained. This has been illustrated on mechanical systems with kinematic constraints and constrained systems on dual Lie algebras. Canonical 
coordinates for (closed) Dirac structures have been discussed, as well as their use for stability analysis of implicit Hamiltonian systems. Finally the theory has been applied to implicit port-controlled generalized Hamiltonian systems, such as actuated mechanical systems with kinematic constraints, and it has been shown in particular that the closedness condition for the Dirac structure leads to strong conditions on the input vector fields.

Acknowledgments. We would like to thank Bernhard Maschke (Conservatoire National des Arts et Métiers, Paris) for stimulating discussions. We also thank Peter Crouch (Arizona State University) and Tony Bloch (University of Michigan) for pointing out to the second author the relevance of Dirac structures.

\section{REFERENCES}

[AKN] V.I. Arnold, V.V. Kozlov, and A.I. Neishtadt, Mathematical Aspects of Classical and Celestial Mechanics, Springer-Verlag, Berlin, New York, 1997.

[AmR] R. Abraham, J.E. Marsden, and T. Ratiu, Manifolds, Tensor Analysis, and Applications, Springer-Verlag, Berlin, New York, 1988.

[BC] A.M. Bloch And P.E. CRouch, Nonholonomic control systems on Riemannian manifolds, SIAM J. Control Optim., 33 (1995), pp. 126-148.

[BKMM] A.M. Bloch, P.S. Krishnaprasad, J.E. Marsden, and R.M. Murray, Nonholonomic mechanical systems with symmetry, Arch. Rational Mech. Anal., 136 (1996), pp. 21-99.

[C1] T.J. Courant, Dirac manifolds, Trans. Amer. Math. Soc., 319 (1990), pp. 631-661.

[C2] P.E. Crouch, Handwritten notes, 1994.

[CW] T.J. Courant And A. Weinstein, Beyond Poisson structures, in Seminaire sudrhodanien de geometrie 8, Travaux en Cours 27, Hermann, Paris, 1988.

[D1] I. Dorfman, Dirac structures of integrable evolution equations, Phys. Lett. A, 125 (1987), pp. 240-246.

[D2] I. Dorfman, Dirac Structures and Integrability of Nonlinear Evolution Equations, John Wiley, Chichester, 1993.

[D3] P. Dirac, Generalized Hamiltonian dynamics, Canad. J. Math., 2 (1950), pp. 129-148.

[JE] D.L. Jones And F.J. Evans, Variational analysis of electrical networks, J. Franklin Inst., 295 (1973), pp. 9-23.

[M1] A.G.J. MACFARLANe, An integral formulation of a canonical equation set for non-linear electrical networks, Internat. J. Control, 11 (1970), pp. 449-470.

[M2] B.M. Maschke, Elements on the modelling of multibody systems, in Modelling and Control of Mechanisms and Robot Systems, C. Melchiorri and A. Tornambe, eds., World Scientific Publishing Ltd., River Edge, NJ, 1996, pp. 1-38.

[MBS] B.M. MASchKe, C. Bidard, AND A.J. VAN DER Schaft, Screw-vector bond graphs for the kinestatic and dynamic modeling of multibody systems, Dynamic Systems and Control, 55(1994), pp. 637-644.

[MR] J.E. MARSDen AND T. RATIU, Introduction to Mechanics and Symmetry, SpringerVerlag, Berlin, New York, 1994.

[MS1] B.M. Maschke ANd A.J. VAn Der Schaft, Port-controlled Hamiltonian systems: Modelling origins and system theoretic properties, in Proc. IFAC Symp. NOLCOS, Bordeaux, France, 1992, M. Fliess, ed., International Federation of Automatic Control, pp. $282-288$.

[MS2] B.M. MASChKE AND A.J. VAN DER SCHAFT, System-theoretic properties of port-controlled Hamiltonian systems, in Systems and Networks: Mathematical Theory and Applications, vol. II, Akademie Verlag, Berlin, 1994, pp. 349-352.

[MS3] B.M. MASChKe AND A.J. van DER Schaft, A Hamiltonian approach to stabilization of nonholonomic mechanical systems, in Proc. 33rd IEEE CDC, Orlando, FL, 1994, IEEE Control Systems Society, pp. 2950-2954.

[MSB1] B.M. Maschke, A.J. van Der Schaft, And P.C. Breedveld, An intrinsic Hamiltonian formulation of network dynamics: Non-standard Poisson structures and gyrators, J. Franklin Inst., 329 (1992), pp. 923-966. 
[MSB2] B.M. Maschke, A.J. van Der Schaft, And P.C. Breedveld, An intrinsic Hamiltonian formulation of the dynamics of LC-circuits, IEEE Trans. Circuits Systems I Fund. Theory Appl., 42 (1995), pp. 73-82.

[NS] H. NiJmeiJer AND A.J. van Der Schaft, Nonlinear Dynamical Control Systems, Springer-Verlag, Berlin, New York, 1990.

[O] P.J. Olver, Applications of Lie Groups to Differential Equations, 2nd ed., SpringerVerlag, Berlin, New York, 1993.

[OS] R. Ortega And M.W. Spong, Adaptive motion control of rigid robots: A tutorial, Automatica J. IFAC, 25 (1989), pp. 877-888.

[S] J.J. Slotine, Putting Physics in Control: The Example of Robotics, IEEE Control Systems Mag., 8 (1988), pp. 12-18.

[SM1] A.J. VAn DER SChAFT AND B.M. MASchKe, On the Hamiltonian formulation of nonholonomic mechanical systems, Rep. Math. Phys., 34 (1994), pp. 225-233.

[SM2] A.J. VAN DER SCHAFT AND B.M. MASChKe, The Hamiltonian formulation of energy conserving physical systems with external ports, Arch. für Elektronik und Übertragungstechnik, 49 (1995), pp. 362-371.

[SM3] A.J. VAN DER SCHAFT AND B.M. MASChKe, Mathematical modelling of constrained Hamiltonian systems, in Proc. IFAC Symp. NOLCOS, Tahoe City, CA, 1995, International Federation of Automatic Control, pp. 678-683.

[TA] M. Takegaki and S. ARimoto, A new feedback method for dynamic control of manipulators, Trans. ASME J. Dynam. System Meas. Control, 103 (1981), pp. 119-125.

[W] A. Weinstein, The local structure of Poisson manifolds, J. Differential Geom., 18 (1983), pp. $523-557$. 